\title{
IMPROVED $\log (g f)$ VALUES FOR LINES OF Ti I AND ABUNDANCE DETERMINATIONS IN THE PHOTOSPHERES OF THE SUN AND METAL-POOR STAR HD 84937 (ACCURATE TRANSITION PROBABILITIES FOR Ti I)
}

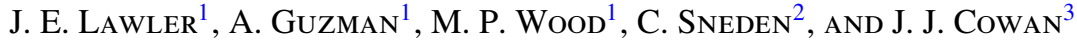 \\ ${ }^{1}$ Department of Physics, University of Wisconsin, Madison, WI 53706, USA; jelawler@wisc.edu, adrianaguzman2014@u.northwestern.edu, mpwood@wisc.edu \\ ${ }^{2}$ Department of Astronomy and McDonald Observatory, University of Texas, Austin, TX 78712, USA; chris@ verdi.as.utexas.edu \\ ${ }^{3}$ Homer L. Dodge Department of Physics and Astronomy, University of Oklahoma, Norman, OK 73019, USA; cowan@nhn.ou.edu \\ Received 2012 November 28; accepted 2013 January 28; published 2013 March 5
}

\begin{abstract}
New atomic transition probability measurements for 948 lines of Ti I are reported. Branching fractions from Fourier transform spectra and from spectra recorded using a $3 \mathrm{~m}$ echelle spectrometer are combined with published radiative lifetimes from laser-induced fluorescence measurements to determine these transition probabilities. Generally good agreement is found in comparisons to the NIST Atomic Spectra Database. The new TiI data are applied to re-determine the Ti abundance in the photospheres of the Sun and metal-poor star HD 84937 using many lines covering a range of wavelength and excitation potential to explore possible non-local thermal equilibrium effects. The variation of relative $\mathrm{Ti} / \mathrm{Fe}$ abundance with metallicity in metal-poor stars observed in earlier studies is supported in this study.
\end{abstract}

Key words: atomic data - methods: laboratory - stars: abundances

Online-only material: color figures, machine-readable tables

\section{INTRODUCTION}

Atomic transition probabilities for the first and second spectra of iron (Fe)-group elements needed for stellar element abundance studies are fairly extensive, for example, in the NIST Atomic Spectra Database ${ }^{4}$ and in the Vienna Atomic Line Database. ${ }^{5}$ The resulting abundances and their trends as a function of metallicity $([\mathrm{Fe} / \mathrm{H}])$ give important information regarding the astrophysical origin and nucleosynthetic history of chemical elements in the Galaxy. ${ }^{6}$ The available lab data are much improved by the use of modern experimental and theoretical methods, such as laser-induced fluorescence (LIF) for radiative lifetime measurements which are combined with emission branching fractions from data recorded with a powerful Fourier transform spectrometer (FTS). In spite of these advances, recent studies have found unexpected trends in relative values of $\mathrm{Fe}-$ group element abundances versus metallicity in metal-poor stars (McWilliam et al. 1995a, 1995b; McWilliam 1997; Westin et al. 2000; Cowan et al. 2002; Sneden et al. 2003; Cayrel et al. 2004; Barklem et al. 2005). The $[\mathrm{X} / \mathrm{Fe}]$ trends range covers \pm 1 dex for metallicity $[\mathrm{Fe} / \mathrm{H}]$ values from 0 to -4 (e.g., Figure 12 of McWilliam 1997). These results have not been reconciled with current models of supernova yields in the early Galaxy.

In earlier decades, it was common to dismiss such anomalies as due to inaccuracies of the atomic transition probabilities. Order of magnitude (1 dex) errors in experimental atomic transition probabilities are now quite rare. However, there is still some possibility that inaccuracies of atomic transition probabilities are contributing to the unexpected Fe-group abundance trends. In studies covering a wide range of metallicity, $>2$ dex, it is necessary to change from weak lines with large excitation

\footnotetext{
4 Available at http://physics.nist.gov/PhysRefData/ASD/lines_form.html and http://physics.nist.gov/cgi-bin/ASBib1/TransProbBib.cgi.

5 Available at http://www.astro.uu.se/ vald/php/vald.php.

6 We adopt standard spectroscopic notations. For element X, the "absolute" abundance is written $\log \varepsilon(\mathrm{X})=\log _{10}\left(N_{\mathrm{X}} / N_{\mathrm{H}}\right)$. For elements $\mathrm{X}$ and $\mathrm{Y}$, the relative abundances are written $[\mathrm{X} / \mathrm{Y}]=\log _{10}\left(N_{\mathrm{X}} / \mathrm{N}_{\mathrm{Y}}\right)_{\mathrm{star}}-\log _{10}\left(N_{\mathrm{X}} / N_{\mathrm{Y}}\right)_{\odot}$. Metallicity will be considered equivalent to the $[\mathrm{Fe} / \mathrm{H}]$ value.
}

potential (E.P.) values in the first spectra (neutral atoms are a minor ionization stage in stars of interest) to stronger lines with smaller E.P. values and eventually to lines of the second spectra (singly ionized atoms are the dominant ionization stage). The need to use so many different lines in studies covering large metallicity ranges creates a possibility that uncertainties of atomic transition probabilities can still affect outcomes. There is also a possibility that the production of Fe-group elements in the early universe is not as well understood as generally believed. A third possibility is that traditional one-dimensional/local thermodynamic equilibrium (1D/LTE) photospheric models are failing in metal-poor stars (e.g., Asplund 2005). Giant stars are favored in studies of metal-poor stars to provide the photon flux needed for high signal-to-noise $(\mathrm{S} / \mathrm{N})$ spectra with high resolution. Low collision rates inherent to the low-density atmospheres of giant stars are further suppressed by the low electron pressures from the low metal content in stars of interest. Setting aside the possible second (nuclear physics) explanation for the unexpected $\mathrm{Fe}$-group trends, the first and third possible explanations can be addressed using further improvements in atomic transition probabilities of Fe-group spectra. If 3D/non-LTE effects in giant metal-poor stars are the cause of the trends, an empirical approach of mapping anomalous abundance results for various spectral lines in a wide range of stellar types is a way forward. Although this approach is time consuming, the alternative approach of incorporating $3 \mathrm{D} /$ non-LTE effects in photospheric models is also quite difficult. A major challenge of non-LTE modeling is the lack of key atomic data, such as cross sections or rate constants for inelastic collisions of ground level $\mathrm{H}$ and He atoms with ground level and excited metal atoms and ions (e.g., Asplund 2005).

An effort is underway to revisit the Fe-group and "squeeze down" uncertainties of selected first and second spectra lines (e.g., Den Hartog et al. 2011). The work on Mn I and Mn II by Den Hartog et al. focused on sets of lines useful for abundance determinations and covering a range of wavelength and a range of E.P. values in both spectra. Because the multiplets of interest individually covered small wavelength ranges and/or 
are Russell-Saunders (LS) multiplets, it was possible to reduce the $\log (g f)$ uncertainties to 0.02 dex with $2 \sigma$ confidence. Such small uncertainties in branching fraction measurements are difficult to achieve and typically are practical only under favorable circumstances. In any case such small $0.01 \mathrm{dex}(1 \sigma)$ uncertainties are not essential for detecting non-LTE effects in metal-poor stars. Manganese is an element of interest for studies of non-LTE effects (Bergemann \& Gehren 2007, 2008). Non-LTE effects of 0.5-1 dex found using the Mn I and Mn II data from Den Hartog et al. on a selected set of metal-poor stars are confined to Mn I resonance lines (connected to the ground level; J. S. Sobeck et al. 2013, in preparation). In this work on Ti I, we use a broader approach and measure emission branching fractions for every possible line connected to the 146 odd-parity upper levels with lifetime measurements by Salih \& Lawler (1990) and Lawler (1991). Branching fractions for lines from the 15 even-parity upper levels studied by Salih \& Lawler were reported earlier (Nitz et al. 1998), but the E.P. values of these lines are too high for most abundance determinations. The resulting set of $948 \log (g f)$ values for lines from odd-parity upper levels covers a wide range of wavelength and E. P. in Ti I. Each $\log (g f)$ is normalized using a radiative lifetime measurement from the same LIF experiment. Uncertainties in the $\log (g f)$ values range from 0.02 dex for dominant branches to $\sim 0.10 \mathrm{dex}$ for weak branches widely separated from the dominant branch or branches. The 0.02 dex uncertainty of dominant branches is primarily from the LIF measurements.

The new Ti I data are applied to re-determine the Ti abundance in the photospheres of the Sun and metal-poor star HD 84937 using many lines covering a range of wavelength and E. P. values to explore possible non-LTE effects. Only a rather small effect is found and it is localized at wavelengths near the Balmer limit. The effect may be due to overpopulation of the $n=2$ levels of $\mathrm{H}$. The variation observed in earlier studies of $[\mathrm{Ti} / \mathrm{Fe}]$ with metallicity in metal-poor stars is supported in this study.

\section{FOURIER TRANSFORM SPECTROMETER DATA}

As discussed above, radiative lifetimes from Salih \& Lawler (1990) and Lawler (1991) provide the absolute normalization for this study of branching fractions and transition probabilities of Ti I. Branching fraction measurements are attempted on lines from all 146 odd-parity levels of the lifetime experiments and are completed for 124 odd-parity upper levels. As in much of our earlier work on branching fractions, we use data from the $1 \mathrm{~m}$ FTS at the National Solar Observatory (NSO) on Kitt Peak for this study of Ti I branching fractions (Brault 1976). The advantages of an FTS include: a large etendue, a high resolving power, an exceptional wavenumber accuracy, a broad spectral coverage, and a high data collection rate.

Multiple FTS spectra are needed to determine high-quality branching fractions. Optimum sensitivity is achieved in different spectral ranges using different detectors and optical components such as the FTS beam splitter. A range of lamp currents is also needed to measure accurate branching ratios. Strong lines which are prone to optical depth errors must be measured at low lamp currents and weak lines must be measured at high lamp currents to achieve good $\mathrm{S} / \mathrm{N}$. Table 1 is a list of the 17 FTS spectra used in our Ti I branching fraction study. All 17 spectra, raw interferograms, and header files are available in the electronic archives of the National Solar Observatory. ${ }^{7}$ Some $\mathrm{Ti} / \mathrm{Ne}$ hollow cathode spectra are included so that a few blends

\footnotetext{
Available at http://nsokp.nso.edu/.
}

of Ti I lines with Ar lines can be corrected. Most of these archived spectra are from lamps at rather high operating currents. Such spectra are ideal for measuring branching fractions of many weak lines, but most of these spectra have non-negligible optical depth problems on strong lines. Additional Ti hollow cathode discharge (HCD) lamp spectra recorded with a new echelle spectrometer are described in the next section. The additional Ti spectra recorded with the echelle spectrometer are used to address the optical depth problems.

Emission branching fraction measurements do not require an absolute radiometric calibration of the spectrometer, but an accurate relative radiometric calibration is essential. The Ar I and Ar II line calibration technique is often used to calibrate FTS instruments and we use that technique to calibrate the spectra in Table 1. Well-known branching ratios for sets of Ar I and Ar II lines widely separated in wavelength (Adams \& Whaling 1981; Danzmann \& Kock 1982; Hashiguchi \& Hasikuni 1985; Whaling et al. 1993) are compared to the intensities measured for the same lines to construct a relative radiometric calibration. This technique captures the wavelengthdependent response of detectors, spectrometer optics, lamp windows, and other components in the light path or any reflections which contribute to the detected signal. The additional Ti spectra recorded with the echelle spectrometer described in the next section are radiometrically calibrated using continuum standard lamps including a NIST traceable $\mathrm{D}_{2}$ lamp and a NIST calibrated Ar mini-arc. These additional calibrated spectra are used to correct the optical depth problems in the FTS data. The echelle spectra also provide good branching fraction measurements on Ti I lines down to $2300 \AA$ since they are calibrated to much shorter wavelengths.

\section{ECHELLE SPECTROMETER DATA}

Operation of the NSO $1 \mathrm{~m}$ FTS on Kitt Peak has been terminated. The instrument has been disassembled and removed from the Observatory. There are prospects that it will be restored to full operation in a university lab, but it is not currently available to NSO guest observers. This development provides one motivation for developing a powerful echelle spectrometer at the University of Wisconsin.

The desire to map non-LTE effects on Fe-group abundance determinations in metal-poor stars provides another motivation for developing a new echelle spectrometer for lab astrophysics. Lines connecting to the ground and low metastable levels (if such levels exist) of Fe-group ions are relatively insensitive to abundance errors from non-LTE effects. Although there is some concern that resonance scattering in the line can perturb the LTE opacity and/or source function, the lower level populations are in LTE. This is because $>90 \%$ of the Fe-group atoms are singly ionized in the photospheres of metal-poor stars of interest, and $\geqslant 75 \%$ of the Fe-group ions are in the ground or low metastable levels (if such levels exist). It is perfectly reasonable that a minor ionization stage or very slightly populated level in the dominant ionization stage can be out of equilibrium. However, since the ground and near ground levels of the ion are the primary population reservoir, these levels cannot be significantly out of equilibrium. This simple realization from a Boltzmann/Saha analysis means that UV lines connecting to ground and low metastable levels of $\mathrm{Fe}$-group ions are the "gold standard" for mapping non-LTE effects in metal-poor stars. Accurate absolute transition probabilities for second spectra lines, especially for weak lines, connecting to those low levels are thus very important. Preferred second spectra Fe-group lines 
Table 1

Fourier Transform Spectra of a Custom Water-cooled Ti Hollow Cathode Discharge (HCD) Lamp

\begin{tabular}{|c|c|c|c|c|c|c|c|c|c|c|c|}
\hline Index & Date & Serial Number & Lamp Type & Buffer Gas & $\begin{array}{l}\text { Lamp Current } \\
\text { (mA) }\end{array}$ & $\begin{array}{l}\text { Wavenumber Range } \\
\qquad\left(\mathrm{cm}^{-1}\right)\end{array}$ & $\begin{array}{l}\text { Limit of Resolution } \\
\qquad\left(\mathrm{cm}^{-1}\right)\end{array}$ & Co-adds & Beam Splitter & Filter & Detector $^{\mathrm{a}}$ \\
\hline 1 & 1991 Feb 21 & 4 & Custom HCD & $\mathrm{Ar}-\mathrm{Ne}$ & 240 & $7972-41160$ & 0.042 & 8 & UV & & Mid Range Si Diode \\
\hline 2 & 1989 Mar 9 & 16 & Custom HCD & $\mathrm{Ar}$ & 770 & 7404-33520 & 0.042 & 3 & UV & WG295 & Super Blue Si Diode \\
\hline 3 & 1989 Mar 14 & 18 & Custom HCD & $\mathrm{Ar}$ & 900 & $7673-34328$ & 0.042 & 6 & UV & WG295 & Super Blue Si Diode \\
\hline 4 & 1991 May 1 & 4 & Custom HCD & $\mathrm{Ar}$ & 434 & $8460-43317$ & 0.050 & 6 & UV & $\mathrm{CuSO}_{4}$ & Mid Range Si Diode \\
\hline 5 & 1991 May 3 & 16 & Custom HCD & $\mathrm{Ar}$ & 150 & $8460-43317$ & 0.050 & 6 & UV & $\mathrm{CuSO}_{4}$ & Mid Range Si Diode \\
\hline 6 & 1991 May 3 & 17 & Custom HCD & $\mathrm{Ar}$ & 610 & $8460-43317$ & 0.050 & 6 & UV & $\mathrm{CuSO}_{4}$ & Mid Range Si Diode \\
\hline 7 & 1989 Feb 28 & 25 & Custom HCD & $\mathrm{Ar}$ & 820 & $8368-36310$ & 0.042 & 6 & UV & WG295 & Super Blue Si Diode \\
\hline 8 & 1992 Jul 28 & 1 & Custom HCD & $\mathrm{Ar}$ & 410 & $8334-45394$ & 0.046 & 8 & UV & WG230 & Mid Range Si Diode \\
\hline 9 & 1992 Jul 29 & 2 & Custom HCD & $\mathrm{Ar}$ & 140 & $8421-45507$ & 0.046 & 2 & UV & WG230 & Mid Range Si Diode \\
\hline 10 & 1992 Jul 29 & 3 & Custom HCD & $\mathrm{Ar}$ & 145 & $8421-45507$ & 0.046 & 4 & UV & WG230 & Mid Range Si Diode \\
\hline 11 & 1992 Jul 29 & 4 & Custom HCD & $\mathrm{Ar}$ & 148 & $8421-45865$ & 0.046 & 8 & UV & WG230 & Mid Range Si Diode \\
\hline 12 & 1991 May 1 & 1 & Custom HCD & $\mathrm{Ne}$ & 53 & $8460-43317$ & 0.050 & 6 & UV & $\mathrm{CuSO}_{4}$ & Mid Range Si Diode \\
\hline 13 & 1991 May 1 & 2 & Custom HCD & $\mathrm{Ne}$ & 102 & $8460-43317$ & 0.050 & 6 & UV & $\mathrm{CuSO}_{4}$ & Mid Range Si Diode \\
\hline 14 & 1991 May 1 & 3 & Custom HCD & $\mathrm{Ne}$ & 202 & $8460-43317$ & 0.050 & 6 & UV & $\mathrm{CuSO}_{4}$ & Mid Range Si Diode \\
\hline 15 & $1991 \mathrm{Feb} 20$ & 1 & Custom HCD & $\mathrm{Ar}-\mathrm{Ne}$ & 310 & $3300-22206$ & 0.023 & 4 & UV & GG $475 \& \mathrm{Si}$ & Mid Range Si Diode \& InSb \\
\hline 16 & $1991 \mathrm{Feb} 20$ & 2 & Custom HCD & $\mathrm{Ar}-\mathrm{Ne}$ & 207 & $3300-22206$ & 0.023 & 2 & UV & GG $475 \& \mathrm{Si}$ & Mid Range Si Diode \& InSb \\
\hline 17 & 1991 Feb 20 & 3 & Custom HCD & $\mathrm{Ar}-\mathrm{Ne}$ & 510 & $3300-22206$ & 0.023 & 2 & UV & GG $475 \& \mathrm{Si}$ & Mid Range Si Diode \& InSb \\
\hline
\end{tabular}

Notes. All were recorded using the $1 \mathrm{~m}$ FTS on the McMath telescope at the National Solar Observatory, Kitt Peak, AZ.

${ }^{a}$ Detector types include the super blue silicon ( $\mathrm{Si}$ ) photodiode, mid range Si photodiode, and an indium antimonide (InSb) detector for the near-infrared. 
Table 2

Echelle Spectra of Commercial Ti HCD Lamps and a Custom Water-cooled Ti HCD Lamp

\begin{tabular}{|c|c|c|c|c|c|c|c|c|c|}
\hline Index & Date & Serial Numbers ${ }^{\mathrm{a}}$ & Lamp Type ${ }^{b}$ & Buffer Gas & $\begin{array}{l}\text { Lamp Current } \\
\qquad(\mathrm{mA})\end{array}$ & $\begin{array}{c}\text { Wavelength Range } \\
(\AA)\end{array}$ & Resolving Power & Co-adds & $\begin{array}{c}\text { Exposure Time } \\
\text { (s) }\end{array}$ \\
\hline $21-23$ & 2011 Jul 27 & $4,8,12$ & Commercial HCD & $\mathrm{Ne}$ & 10 & $2200-3900$ & 250,000 & 10 & 360 \\
\hline $24-26$ & 2011 Jul 28 & $4,8,12$ & Commercial HCD & $\mathrm{Ne}$ & 15 & $2200-3900$ & 250,000 & 10 & 360 \\
\hline $27-29$ & 2011 Aug 12 & $4,8,12$ & Commercial HCD & $\mathrm{Ne}$ & 7 & $2200-3900$ & 250,000 & 20 & 180 \\
\hline $30-32$ & 2011 Aug 9 & $4,8,12$ & Commercial HCD & $\mathrm{Ar}$ & 10 & $2200-3900$ & 250,000 & 10 & 360 \\
\hline $33-35$ & 2011 Sep 1 & $4,8,12$ & Custom HCD & $\mathrm{Ar}$ & 25 & $2200-3900$ & 250,000 & 20 & 120 \\
\hline $36-38$ & 2011 Aug 30 & $4,8,12$ & Custom HCD & $\mathrm{Ar}$ & 40 & $2200-3900$ & 250,000 & 20 & 60 \\
\hline $39-43$ & 2011 Sep 22 & $2,5,9,12,16$ & Commercial HCD & $\mathrm{Ar}$ & 10 & $2200-3900$ & 250,000 & 10 & 180 \\
\hline $44-48$ & 2011 Sep 29 & $2,5,9,12,16$ & Commercial HCD & $\mathrm{Ar}$ & 10 & $2200-3900$ & 250,000 & 10 & 180 \\
\hline $49-53$ & 2011 Oct 24 & $2,5,9,12,16$ & Custom HCD & $\mathrm{Ar}$ & 40 & $2200-3900$ & 250,000 & 20 & 60 \\
\hline $54-58$ & 2011 Nov 4 & $2,5,9,12,16$ & Custom HCD & $\mathrm{Ar}$ & 80 & $2200-3900$ & 250,000 & 20 & 60 \\
\hline $59-63$ & 2012 Feb 21 & $2,5,9,12,16$ & Custom HCD & $\mathrm{Ar}$ & 40 & $2200-3900$ & 250,000 & 40 & 30 \\
\hline $64-68$ & 2012 Feb 23 & $2,5,9,12,16$ & Custom HCD & $\mathrm{Ar}$ & 40 & $2200-3900$ & 250,000 & 40 & 30 \\
\hline
\end{tabular}

Notes.

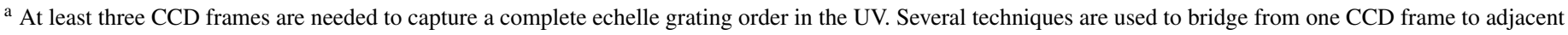

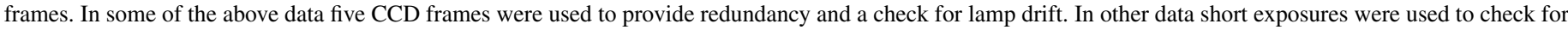
lamp drift.

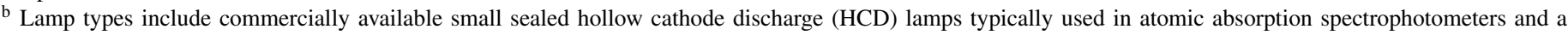
custom water-cooled HCD lamp.

need to be weak to avoid saturation, and they are almost all in the UV. The multiplex noise inherent to all FTS instruments can be a problem when measuring weak UV lines. Typically, both optical and detector efficiencies are higher at longer wavelengths. The smooth redistribution of quantum statistical (Poisson) noise from all spectral features, including the strong visible and near-IR features, is a hindrance in the UV. Sometimes weak UV lines "melt" into the multiplex noise before the dominant lines from the common upper level become optically thin as the lamp current is reduced. Dispersive spectrometers are free from multiplex noise.

The echelle spectrometer incorporates a vacuum compatible McPherson model $21733 \mathrm{~m}$ focal length Czerny-Turner grating spectrograph which was previously used to measure relative strengths of UV and vacuum-UV branches in support of investigations of the interstellar medium with the Hubble Space Telescope (HST; e.g., Bergeson et al. 1996; Lawler et al. 1999). The standard grating is replaced with a large $(128 \times 254 \mathrm{~mm}$ ruled area), coarse (23.2 grooves $\mathrm{mm}^{-1}$ ) echelle grating blazed at 63.5. The entrance slit assembly is modified to accept precision machined pinholes of various diameters. Attached to the exit port of the grating spectrograph is a custom built $0.5 \mathrm{~m}$ focal length prismatic order separator, rotated into a plane orthogonal to the grating spectrograph, that serves to separate the many overlapping high orders from the coarse echelle grating into a 2D spectrum suitable for imaging onto a square $(2048 \times 2048,13.5 \mu \mathrm{m}$ square pixels $)$ UV sensitive CCD array (Princeton Instruments PI-SX:2048). The plane rotation serves to cancel the astigmatism that is the dominant aberration inherent in the main grating spectrometer. The complete echelle instrument has a resolving power of 250,000 with a $50 \mu \mathrm{m}$ diameter entrance pinhole, broad spectral coverage with a few CCD frames, superb UV sensitivity, and great flexibility from the UV to the silicon detector limit in the IR. The most significant disadvantage compared to an FTS is the lower accuracy and precision of wavelength or wavenumber measurements, and the most significant advantage is the absence of multiplex noise. The design and performance of the instrument, along with an aberration analysis, is described in detail by Wood \& Lawler (2012).
In addition to the 17 FTS spectra of Table 1, the 48 CCD frames of spectra from Ti HCD lamps listed in Table 2 are part of this Ti I branching fraction study. All of the spectra listed in Tables 1 and 2 are also being used to refine branching fraction measurements on Ti II (Wood et al. 2013). Unlike Ti I there are already two sets of published Ti II branching fraction measurements from FTS data (Bizzarri et al. 1993; Pickering et al. 2001). Even with the published work on Ti II some improvement in $\log (g f)$ values is anticipated by comparing results from different spectrometers and radiometric calibration methods. Systematic uncertainties dominate most branching fraction measurements and these uncertainties are difficult to control and reliably estimate.

All of the spectra in Table 2 are radiometrically calibrated using continuum standard lamps including a NIST traceable $\mathrm{D}_{2}$ lamp and a NIST calibrated Ar mini-arc. The use of standard lamps in the UV to calibrate a FTS is often difficult due to ghosts, but it is our preferred method for the echelle spectrometer.

\section{Ti I BRANCHING FRACTIONS}

The $|\Delta L| \leqslant 1$ and $\Delta S=0$ selection rules of LS or Russell-Saunders coupling are violated by many important transitions of Fe-group and heavier species. Because only the parity change and $|\Delta J| \leqslant 1$ selection rules are obeyed throughout the periodic table, all possible transition wavenumbers between known energy levels of Ti I satisfying these selection rules are computed and used during analysis of FTS data. (Transitions violating the parity and/or $\Delta J$ selection rules are suppressed by a factor of $\sim 10^{6}$ or more and are usually not important in stellar spectroscopy.) Energy levels from Saloman (2012) are used to determine possible transition wavenumbers. Using high current spectra we are able to make measurements on branching fractions as weak as 0.0001 and thus systematic errors from missing branches to known lower levels are negligible. Although Ti has five naturally occurring isotopes, two of which have hyperfine structure due to a non-zero nuclear spin, lines of Ti I are rather narrow in FTS data and component structure is neglected in this study. 
Table 3

Experimental Atomic Transition Probabilities for 948 Lines of Ti I from Upper Odd-parity Levels Organized by Increasing Wavelength in Air

\begin{tabular}{lccccc}
\hline \hline $\begin{array}{l}\text { Wavelength in Air } \\
(\AA)\end{array}$ & $\begin{array}{c}\text { Upper Level Energy } \\
\left(\mathrm{cm}^{-1}\right)\end{array}$ & $J$ & $\begin{array}{c}\text { Lower Level Energy }^{\mathrm{b}} \\
\left(\mathrm{cm}^{-1}\right)\end{array}$ & $\begin{array}{c}J \\
\text { Transition Probability } \\
\left(10^{6} \mathrm{~s}^{-1}\right)\end{array}$ & $\begin{array}{c}\log _{10}(g f) \\
2371.9605\end{array}$ \\
\hline 42146.348 & 1 & 0.000 & 2 & $4.9 \pm 0.5$ \\
2378.1524 & 42206.755 & 2 & 170.133 & 3 & $5.2 \pm 0.5$ \\
2384.5193 & 42311.264 & 3 & 386.874 & 4 & $5.2 \pm 0.5$ \\
2431.7858 & 41170.006 & 3 & 0.000 & 2 & $2.9 \pm 0.6$ \\
\hline
\end{tabular}

Notes.

${ }^{a}$ Wavelength values computed from energy levels using the standard index of air from Peck \& Reeder (1972).

b Level energies, parities, and $J$ values are from Saloman (2012).

(This table is available in its entirety in a machine-readable form in the online journal. A portion is shown here for guidance regarding its form and content.)

Branching fraction measurements are completed for lines from 124 odd-parity upper levels of the 146 odd-parity levels studied in the lifetime experiment by Salih \& Lawler (1990) and Lawler (1991). Some of the levels for which branching fractions are not completed have one or more strong branches with possibly severe blending problems. Other levels for which branching fractions are not completed are sufficiently long lived that they may have significant IR branches or are at such high energy that lines from the levels have poor $\mathrm{S} / \mathrm{N}$ in our data. Thousands of possible spectral line observations are studied during the analysis of lines from 124 upper levels in 17 different Ti FTS spectra and the echelle spectra. The use of FTS and echelle spectrometer data covering a large range of lamp current enables us to easily identify and correct potential errors from blends of Ti I lines with $\mathrm{Ar}$ and Ti II lines. An interactive data analysis routine is used to determine the un-calibrated intensities of Ti I lines and selected Ar I and Ar II lines. This routine enables one to set both integration limits and nonzero baselines for numerical integration. Nonzero baselines are needed during analysis of the echelle spectra because they are not corrected for dark signals and are occasionally needed during analysis of the FTS data when a weak line is located on the wing of a much stronger line. Due to unresolved or weakly resolved isotopic and/or hyperfine structure in Ti I lines a simple numerical integration method is used. Nonlinear least-square fitting of line profiles is justified only when the line sub-component structure is either fully resolved in the FTS data or known from independent measurements.

Branching fraction uncertainties depend on the $\mathrm{S} / \mathrm{N}$ of the data, the wavenumber separations of lines from the common upper level, and the strength of the branching fractions. Consider for example a level with one strong line, $\sim 0.99$, and one weak line, $\sim 0.01$. A very rough ( \pm factor of two) measurement of the ratio of these lines yields a branching fraction of 0.99 for the stronger line with an asymmetric uncertainty of $+0.5 \%$, $-1 \%$. Uncertainty in branching fraction measurements always migrates to the weakest lines because branching fractions sum to 1.0 by definition. Furthermore, the accuracy of weaker line intensity measurements tends to be limited by $\mathrm{S} / \mathrm{N}$. Systematic uncertainty in the relative radiometric calibration is significant for lines widely separated from the dominant line(s) of the common upper. This calibration uncertainty is estimated to be $0.001 \% / \mathrm{cm}^{-1}$ (Wickliffe et al. 2000) and it is combined with the standard deviation of measurements from multiple spectra to determine a total uncertainty of branching fractions. Because systematic uncertainties are significant for many weaker lines, it is not practical to define the branching fraction uncertainties as $1 \sigma$ or $2 \sigma$. The use of multiple spectra from different spectrometers with different calibration techniques is important in assessing and controlling systematic uncertainties.

\section{Ti I TRANSITION PROBABILITIES AND COMPARISON TO EARLIER MEASUREMENTS}

Branching fractions from the FTS and echelle spectra are combined with radiative lifetime measurements (Salih \& Lawler 1990; Lawler 1991) to determine absolute transition probabilities for 948 lines of TiI in Table 3. Air wavelengths in the table are computed from energy levels (Saloman 2012) using the standard index of air (Peck \& Reeder 1972).

Some problem lines have been omitted from Table 3 . The effect of problem lines that are too weak to have a good S/N, have uncertain classification, or are too seriously blended to be separated can be detected in Table 3 by summing all transition probabilities from a chosen upper level and comparing the sum to the inverse of the upper level lifetime (Salih \& Lawler 1990; Lawler 1991). Typically, the sum of the transition probabilities is between $90 \%$ and $100 \%$ of the inverse lifetime. Although there is significant fractional uncertainty in the branching fractions for these problem lines, this does not have much effect on the uncertainty of the lines in Table 3. Branching fraction uncertainties are combined in quadrature with lifetime uncertainties to determine the transition probability uncertainties in Table 3.

Figures 1-4 are comparisons of our Ti I results to the NIST Atomic Spectra Database as of 2012 July 19 (see footnote 4). Values from the database are separated by accuracy ratings in our four figures. Figure 1 compares transition probabilities for 31 lines in common from this work to NIST database values with an "A" $(\leqslant 3 \%)$ accuracy rating. Individual error bars on the plotted $\log (g f)$ differences are uncertainties on measurements from this work. The middle dotted horizontal line represents perfect agreement at a logarithmic difference of 0 . The upper and lower dotted lines represent $\pm 3 \%$ differences in $f$-values. The agreement is generally within combined uncertainties with a couple of exceptions. Morton's (2003) compilation is cited as the source of these " $\mathrm{A}$ " rated data in the NIST Atomic Spectra Database. Morton's compiled results for the "A" rated transition probabilities are from very precise absorption measurements by Blackwell et al. (1982a, 1983) normalized using LIF lifetime measurements from multiple experiments.

Figure 2 compares 27 lines in common from our work to database values with a " $B$ " $(\leqslant 10 \%)$ accuracy rating. The error bars and dotted lines have the same meaning as in Figure 1 except that the upper and lower dotted lines represent $\pm 10 \%$ differences in $f$-values. Blackwell-Whitehead et al. (2006) are 


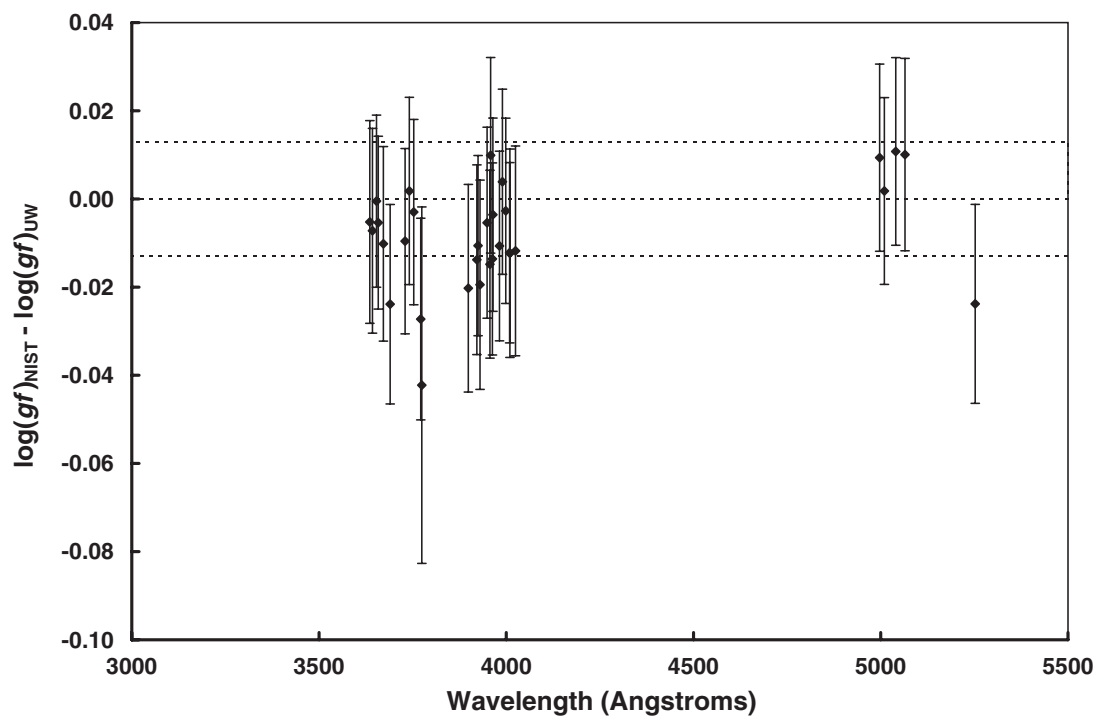

Figure 1. Comparison of our (UW) $\log (g f)$ values for 31 lines to NIST values with accuracy rating " $A$ " $(\leqslant 3 \%)$ in the NIST database. The central dotted line indicates perfect agreement. The upper and lower dotted lines denote $\pm 3 \%$ differences. Error bars on the data points are from our measurements.

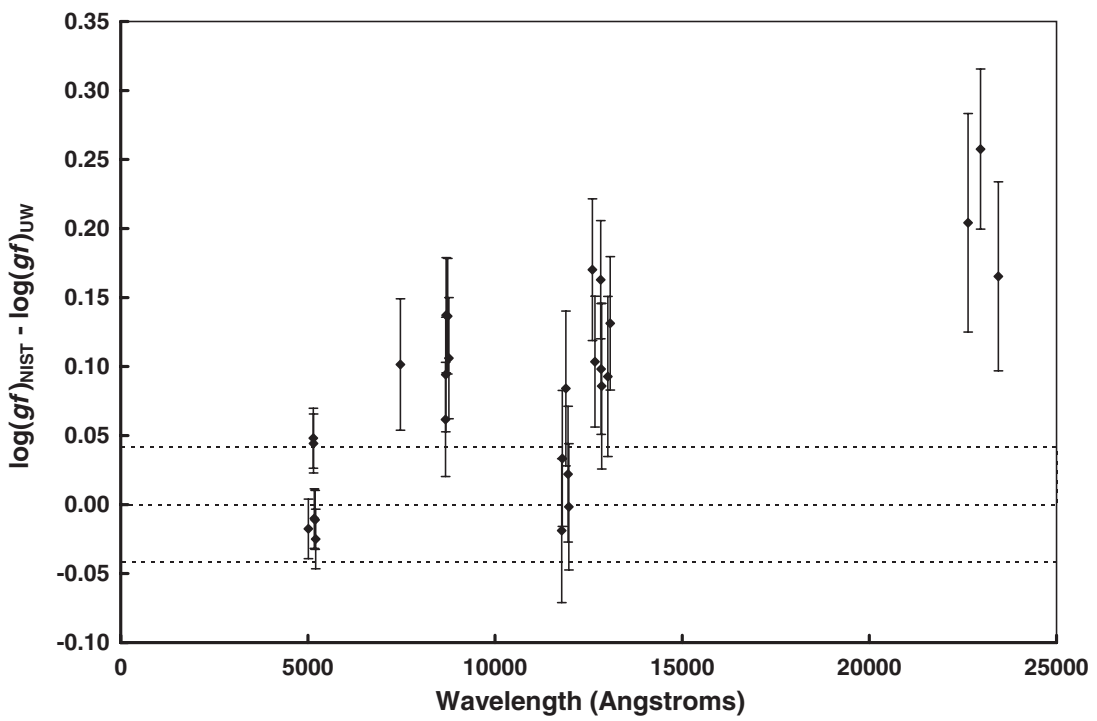

Figure 2. Comparison of our (UW) $\log (g f)$ values for 27 lines to NIST values with accuracy rating " $B$ " $(\leqslant 10 \%)$ in the NIST database. The central dotted line indicates perfect agreement as in Figure 1. The upper and lower dotted lines denote $\pm 10 \%$ differences. Error bars on the data points are from our measurements as in Figure 1.

cited as the source of these " $B$ " rated data in the NIST Atomic Spectra Database. The agreement in Figure 2 is not as good as we expected since Blackwell-Whitehead et al. combined their FTS branching fraction measurements with lifetime measurements from the same sources used in our work (Salih \& Lawler 1990; Lawler 1991). The most serious discordance is for the multiplet between 20000 and $25000 \AA$. These IR lines are minor branches and the discordance is mostly due to a problem in the relative radiometric calibration of one or both branching fraction experiments. Relative radiometric calibrations covering more than a factor of four in wavelength are quite challenging. Blackwell-Whitehead et al. used standard lamps for their relative radiometric calibration and we used the Ar I and Ar II line method. Ar I branching ratios of Groups 31 and 32 in Table 1 of Whaling et al. (1993) provided the extension of our relative radiometric calibration for measurements in the $20000-25000 \AA$ region. The agreement for the other lines is better, but also not quite as good as expected. There is evidence for some optical depth problems in the work by Blackwell-
Whitehead et al. (2006). Points representing differences in $\log (g f)$ of dominant lines around $5000 \AA$ from the three upper levels of interest are about 0.025 dex below the central dotted line in Figure 2. Points for nearby weak lines are above the central dotted line in Figure 2 by more than 0.05 dex. Since radiometric calibration uncertainties are very small over such small wavelength differences, this suppression of strong branches and enhancement of nearby weak branches is likely due to an optical depth problem in the measurements by Blackwell-Whitehead et al. (2006). Elimination of the optical depth problem on the strong branches will bring their $\log (g f)$ values up a small amount and bring the other $\log (g f)$ values for weak branches down by $\sim 0.07$ dex. This correction will result in agreement of 24 of the $27 \log (g f)$ values within combined uncertainties. The weak IR branches between 20000 and $25000 \AA$ will still be in discordance by a bit more than the combined error bars.

The top panel of Figure 3 compares 156 lines in common from our work to NIST database values with a "C" $(\leqslant 25 \%)$ and 

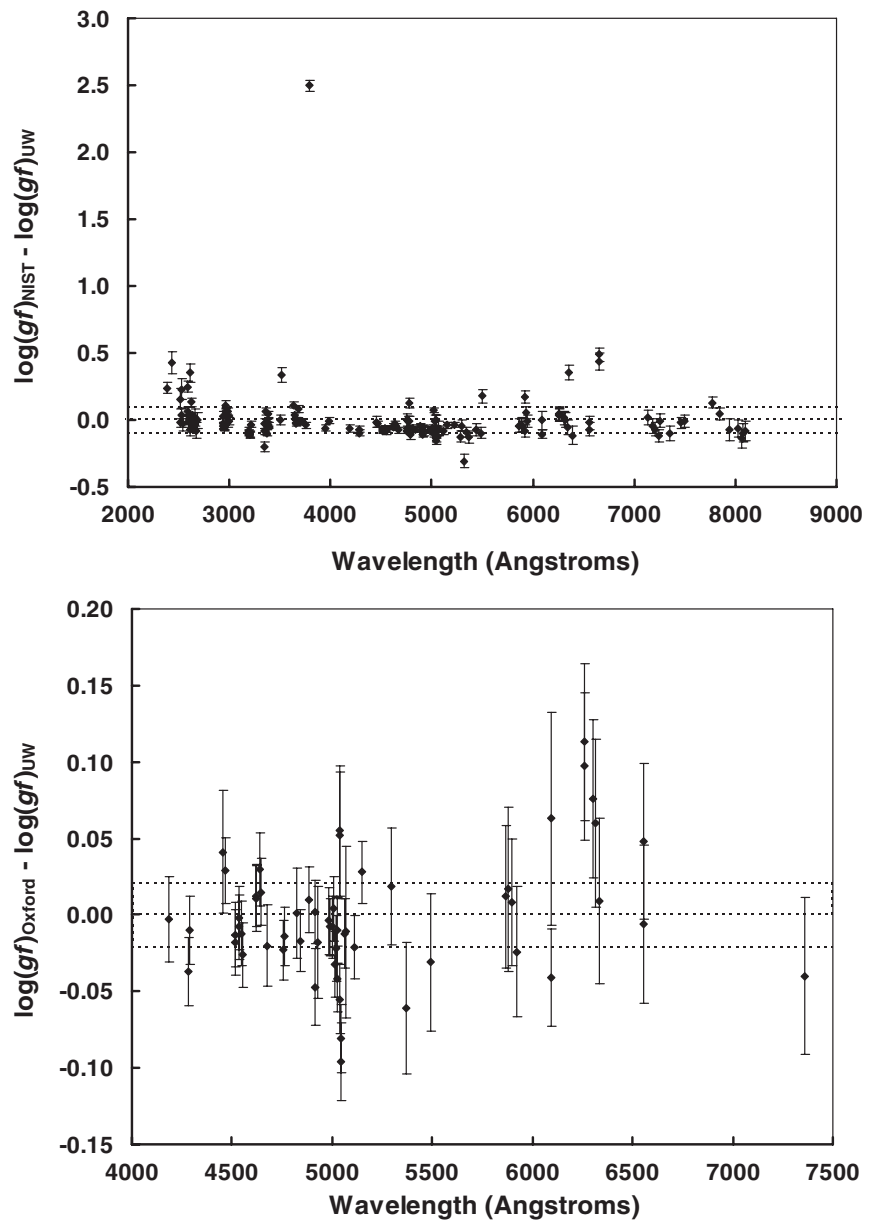

Figure 3. Top panel: comparison of our (UW) $\log (g f)$ values for 156 lines to NIST values with accuracy rating "C" $(\leqslant 25 \%)$ and " $\mathrm{C}+$ " $(\leqslant 18 \%)$ in the NIST database. The central dotted line and error bars on the data points have the same meaning as in Figure 1. The upper and lower dotted lines denote $\pm 25 \%$ differences. Bottom panel: comparison of our (UW) $\log (g f)$ values for 58 lines to renormalized values from the Oxford experiments by Blackwell et al. (1982b, $1983,1986)$. The upper and lower dotted lines denote $\pm 5 \%$ differences.

"C+" ( $\leqslant 18 \%)$ accuracy rating. The error bars and dotted lines have the same meaning as in Figure 1 except that the upper and lower dotted lines represent $\pm 25 \%$ differences in $f$-values. Five sources are cited for the 91 "C" rated data in the NIST Atomic Spectra Database. The lines below $4000 \AA$ are primarily from hook measurements by Smith \& Kühne (1978), but NIST compilers renormalized the transition probabilities. See Martin et al. (1988) for a complete discussion of the renormalization. The lines above $4000 \AA$ are primarily from emission branching fractions by Whaling et al. (1977) normalized using lifetimes measured using the beam foil technique by Roberts et al. (1973). Transition probabilities for two lines, 3354.633 and $3371.452 \AA$, have citations to both Smith \& Kühne (1978) and Bell et al. (1975). Bell et al. used an atomic beam method to measure absolute transition probabilities for a small set of Ti I lines. Transition probabilities for seven lines include citations to Kühne et al. (1978), who used a combination of hook and emission measurements on an arc. Six of those seven lines also have citations to Whaling et al. (1977) or Smith \& Kühne (1978). Transition probabilities for four lines include citations to Holys \& Fuhr (1980) and Whaling et al. (1977). Holys \& Fuhr performed emission measurements on an arc. Five other sources are cited for the 65 "C+" rated transition probabilities in the NIST Atomic Spectra Database. Fifty-eight lines have transition probabilities from absorption measurements by Blackwell et al. (1982b, 1983, 1986). Two lines are from Morton's compilation (2003), and five are from Blackwell-Whitehead et al. (2006). Detailed study of the $156 \log (g f)$ values compared in the top panel of Figure 3 reveals that $\sim 90 \%$ of our measured values agree with the NIST database values within their combined error bars. A few outliers are likely due to misidentified or blended lines in older experiments with lower spectral resolving power. Spectral line widths are much larger in high-pressure ( LTE) arcs than in low-pressure hollow cathode lamps used in our work. Hook measurements have an advantage over absorption measurements in many high-opacity experiments because of much reduced sensitivity to line broadening effects in the analysis of anomalous dispersion data. Hook measurements are challenging in dense spectra because spectral features of interest are spread over substantial wavelength intervals which increases the potential for line blending. The single extreme outlier is the $3788.799 \AA$ spin-forbidden transition from the $y^{5} G^{\circ}{ }_{5}$ upper level to the $a^{3} F_{4}$ level of the ground term and the large transition probability in the NIST database from Morton's compilation is in error.

The lines with "C+" rating by Blackwell et al. (1982b. 1983, and 1986) deserve some extra attention because the Oxford absorption experiment produced relative transition probabilities of exceptional accuracy. Blackwell et al. (1982b) claimed a relative accuracy of $0.5 \%$, Blackwell et al. (1983) claimed relative accuracy of 5\%, and Blackwell et al. (1986) claimed a relative accuracy of $1 \%$. The claimed absolute accuracy of the Oxford measurements was only $15 \%$, but as more LIF data became available on TiI the absolute scale was adjusted. As indicated in the above discussion of Figure 1, NIST compilers adopted the scale adjustment recommended by Morton (2003). Morton added +0.062 dex to the Oxford $\log (g f)$ values and NIST compilers assessed the accuracy of those $\log (g f)$ values in Figure 1 as " $\mathrm{A}$ " $(\leqslant 3 \%)$. Morton's +0.062 renormalization is slightly different than the $+0.056 \mathrm{dex}$ renormalization recommended by Grevesse et al. (1989) based only on Rudolph \& Helbig's LIF lifetime measurements. Salih \& Lawler (1990) compared their lifetimes to those measured by Rudolph \& Helbig (1982) for the nine levels which were used to renormalize the Oxford $\log (g f)$ values and found agreement to within $2.6 \%$ or $0.01 \mathrm{dex}$. In any case the NIST "A" $(\leqslant 3 \%)$ accuracy rating is justified for the low excitation lines from the Oxford measurements included in Morton's compilation. (Morton was only interested in low excitation or "Resonance Absorption Lines" as indicated by his title.) NIST compilers did not renormalize the Oxford $\log (g f)$ values for many higher excitation lines and they simply included those lines with an accuracy rating of " $\mathrm{C}+$ " $(\leqslant 18 \%)$. The bottom panel of Figure 3 includes the 58 "C+" lines from Oxford measurements (Blackwell et al. 1982b, 1983, 1986) with the $\log (g f)$ values adjusted by +0.062 as recommended by Morton. This plot includes many weak lines and many with significant excitation potential. Note that the scale of the vertical or $\log (g f)$ difference axis is expanded by a factor of ten between the top panel and the bottom panel. The bottom panel of Figure 3 supports, to a greater extent than any other plot, the reliability of our uncertainty estimates on $\log (g f)$ values of weaker lines and lines with higher excitation potential.

Figure 4 compares 65 lines in common from our work to database values with a "D" $(\leqslant 50 \%)$ accuracy rating. The error 


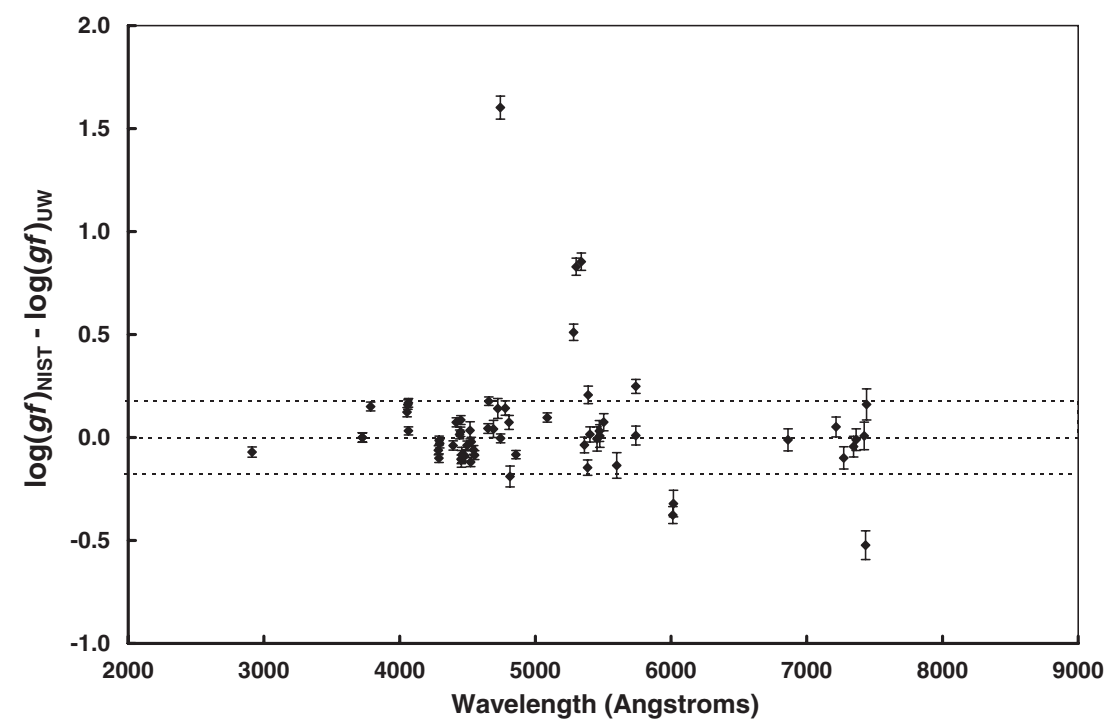

Figure 4. Comparison of our (UW) $\log (g f)$ values for 65 lines to NIST values with accuracy rating " $D$ " $(\leqslant 50 \%)$ in the NIST database. The central dotted line and error bars on the data points have the same meaning as in Figure 1. The upper and lower dotted lines denote $\pm 50 \%$ differences.

bars and dotted lines have the same meaning as in Figure 1 except that the upper and lower dotted lines represent $\pm 50 \%$ differences in $f$-values. Multiple sources are cited for these " $\mathrm{D}$ " rated data in the NIST Atomic Spectra Database. Transition probabilities for 24 lines are from arc emission measurements by Lotrian et al. (1975), but NIST compilers renormalized those emission measurements. Similarly transition probabilities for another 23 lines are from measurements on solar lines by Kostyk (1982), and NIST compilers also renormalized those measurements. Another 10 lines are from Whaling et al. (1977). Transition probabilities for six lines have citations to both Kühne et al. (1978) and Whaling et al. (1977). The transition probability of one of the remaining lines is from Kühne et al. (1978), and another of the remaining lines is from an arc emission measurement by Roberts et al. (1975) with renormalization by NIST compilers. Again $\sim 90 \%$ of the $\log (g f)$ values in common agree within their combined error bars. The outliers which have the largest differences tend to have $\log (g f)$ values well above the values from this experiment.

\section{THE TITANIUM ABUNDANCE IN THE SOLAR PHOTOSPHERE}

We use our new $\mathrm{Ti}$ I transition probabilities to re-determine the solar photospheric $\mathrm{Ti}$ abundance and to generate the first large-line-sample $\mathrm{Ti}$ abundance in the warm very metal-poor star HD 84937. Our analyses generally follow the methods that we employed to derive new accurate abundances of rare-earth elements in a series of papers (Lawler et al. 2009; Sneden et al. 2009, and references therein). In this section, we discuss our solar analyses.

\subsection{Abundance Computation Method and Input Solar Data}

We compute individual synthetic spectra for all transitions studied in the solar spectrum using the current version of the LTE line analysis code MOOG (Sneden 1973). We follow our earlier papers in selection of solar input. The observations are taken from the center-of-disk photospheric spectrum of Delbouille et al. (1973). ${ }^{8}$ For most solar abundance computations we adopt

\footnotetext{
8 Available at http://bass2000.obspm.fr/solar_spect.php.
}

the Holweger \& Müller (1974) empirical model photosphere and use without change the physical quantities as given in the original paper. We compute synthetic spectra in $4 \AA$ intervals surrounding the chosen Ti I transitions. For the needed atomic and molecular line lists we begin with the comprehensive Kurucz (2011, and references therein) database. ${ }^{9}$ We substitute the transition probabilities, and add in hyperfine/isotopic substructure information for neutron-capture elements from our previous papers (Sneden et al. 2009, and references therein). We also adopt recent laboratory transition probabilities for $\mathrm{Cr}$ I from Sobeck et al. (2007), for Y II from Bíemont et al. (2011), and for Zr II from Malcheva et al. (2006). For lines studied in our previous papers and in this paper, we adopt their reported transition probabilities without change. The transition probabilities of other lines are altered to achieve satisfactory matches to the observed solar spectrum. Small wavelength shifts of these lines are also sometimes needed.

\subsection{Ti I Line Selection}

The neutral $\mathrm{Ti}$ species has a complex atomic structure which leads to many potential lines for abundance analyses in solar-type and cooler stars. The Moore et al. (1966) photospheric atlas attributes over 1000 features either partly or entirely due to Ti I absorption. To decide which of these transitions to include here, we first calculated relative strength values for all lines. To first approximation, the relative absorption strengths of lines of a single species in a stellar atmosphere can be written as

$$
\mathrm{STR} \equiv \log (g f)-\theta \chi,
$$

where $\theta$ is the reciprocal temperature $(5040 / T)$ and $\chi$ is the excitation energy. For species that are the dominant contributors to the total elemental abundances and thus have small Saha ionization corrections, the STR data can be compared for different elements by writing STR $\equiv \log (\varepsilon g f)-\theta \chi$, where $\varepsilon$ is the elemental abundance. Such a comparison for Pr II, Dy II, Tm II, and Lu II lines is shown in Figure 1 of Sneden et al. (2009). In the Sun and most metal-poor stars Ti exists predominantly

\footnotetext{
9 http://kurucz.harvard.edu/linelists.html
} 


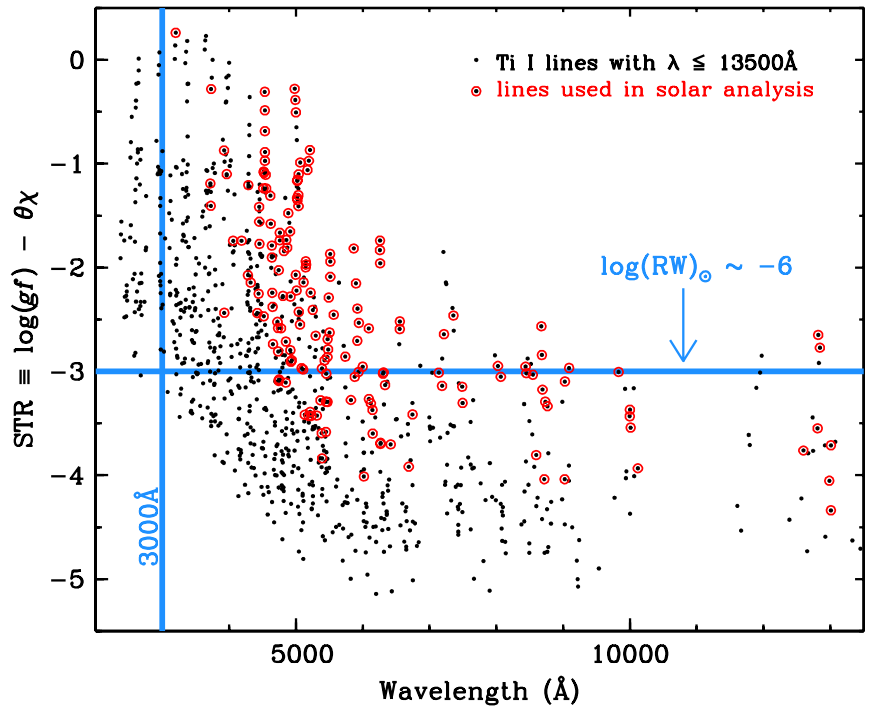

Figure 5. Relative strengths, STR, of Ti I lines as a function of wavelength. Transitions that were used to derive a new solar Ti abundance are marked with "solar" symbols with red borders, as given in the figure legend. There are 19 lines included in the laboratory analysis that lie redward of the plot cutoff at $13,500 \AA$, but these are all very weak and not part of the solar analysis. A blue vertical line at $3000 \AA$ emphasizes the wavelength limit for ground-based spectroscopy. A blue horizontal line indicates the STR level for weak lines, $\log (\mathrm{RW}) \sim-6$.

(A color version of this figure is available in the online journal.)

as Ti II; the strengths of Ti II lines are proportional to the total abundance of Ti.

On the other hand, Ti I (as well as the neutral species of most elements detectable in the solar spectrum and in the spectra of typical A-K stars) comprises only a small fraction of the total Ti abundance. For example, in the solar photosphere at an optical depth $\tau(5000 \AA) \sim 0.5$, with the temperature $T \sim$ $6000 \mathrm{~K}$ and with $\log \left(n_{\mathrm{e}}\right) \sim 13.5$, where $n_{\mathrm{e}}$ is the electron density in $\mathrm{cm}^{-3}$, one finds a Saha ionization ratio $N($ Ti I $) / N($ Ti II $) \sim$ 0.005 . The number density of $\mathrm{Ti}$ and of similar ion-dominated elements are sensitive functions of the ionization energies, and relative strength measures involving the neutral species are difficult to compute without full-scale atmospheric analyses. Therefore, our line strength estimates considered only the Ti I species. For simplicity we assumed a reciprocal temperature $=1.0$ (typical of line-forming regions in G-K stars). We show resulting STR values in Figure 5 as a function of wavelength, encompassing the range to $2000 \AA<\lambda<13,500 \AA$. This range includes the shortest-wavelength Ti I line of our laboratory study (2371 A; Table 3), and the longest-wavelength line used in the solar abundance study (13011 $)$ ). There are 19 lines with new transition probabilities at longer wavelengths, but these are uniformly very weak in the Sun $(\operatorname{STR}<-3.4)$ and will be so in most stars.

The data of Figure 5 show the same trend seen in similar plots for other species in our earlier papers: sharply increasing line strengths toward smaller wavelengths. The simplest indicator of line strength in an observed spectrum is the total absorption expressed as an equivalent width (EW). In optical spectral regions of most stars, the nearly wavelength-independent form of the equivalent width is the reduced width: $\mathrm{RW}=\mathrm{EW} / \lambda$. We make a preliminary survey of detectable but very weak Ti I lines in the Delbouille et al. (1973) photospheric spectrum. This exercise suggests for the Sun, $\log (\mathrm{RW}) \sim-6$ (e.g., EW $\sim 5 \mathrm{~m} \AA$ at $\lambda=5000 \AA$ ) for line strength values STR $\sim-3$. We mark this very weak-line strength level in Figure 5. Further exploration of line detection/measurement limits for very weak lines here and in our past papers suggests that reliable abundances can be derived for lines with $\log (\mathrm{RW}) \geqslant-6.7$ (given the very high $\mathrm{S} / \mathrm{N}$ and resolving power of the Delbouille et al. spectrum), but line detection efforts have little chance of success below this limit. Therefore, we eliminate nearly all potential Ti I lines with STR $<-4$, about 180 lines (nearly $20 \%$ of the original laboratory list of 948 lines), from further consideration.

We then follow the procedures of our previous papers in identifying potentially useful solar Ti I transitions from the remaining lines. We examine small wavelength regions of the Delbouille et al. (1973) center-of-disk spectrum, looking for relatively unblended transitions that could plausibly be attributed to Ti I. Whenever possible we are guided in this effort by the Moore et al. (1966) solar line identifications. Through this process we eliminate Ti I transitions whose photospheric absorptions are undetectably weak and/or severely compromised by blends from other atomic and molecular species. In the end we are left with $\sim 190$ lines for possible solar Ti abundance analysis.

\subsection{Derivation of the Solar Ti Abundance}

As discussed in Section 6.1, we compute synthetic spectra for all 190 lines that survived the elimination process described in Section 6.2, and compare the computed spectra to the Delbouille et al. (1973) solar spectrum. We produce multiple syntheses with different assumed $\mathrm{Ti}$ abundances and smoothed them with Gaussian broadening functions to match the combined effects of spectrograph instrumental profile and solar macro turbulence (the spectrograph is a negligible contributor to the empirical line broadening given the very high resolving power, $R=\lambda / \Delta \lambda>5 \times 10^{5}$ ). In Figure 6 , we show examples of the observed/synthetic spectral matches. In panel (a) one of the longest wavelength, weakest Ti I lines is shown. Neither the very small contamination due to $\mathrm{CN}$ at $12600.16 \AA$ nor the much deeper absorption at the red edge of the panel (due to a strong telluric $\mathrm{H}_{2} \mathrm{O}$ line and a possible solar Fe I line) create much difficulty in deriving a reliable abundance from the Ti I feature. In panel (b) we show another weak Ti I line in the yellow spectral range. The Ti II line at $5454 \AA$ is ignored in this Ti I study. In panel (c) the depressed and sloping "continuum" is due to absorption in the far red wing of the $\mathrm{Mg}_{\mathrm{I}} \mathrm{b}$ line at $5172.7 \AA$, but we derive an abundance from this strong Ti I line that is consistent with other features. Finally, in panel (d) we show one of the best Ti I lines on the low-wavelength end of the transitions that are suitable for solar abundance analysis. The feature at $4060 \AA$ arises from Ti I, but it is not included in our laboratory analyses and should be ignored.

After considering the synthetic and observed spectra in detail, we have a final set of $168 \mathrm{Ti}$ I lines that provide reliable solar Ti abundances. Their parameters and abundance results are given in Table 4. These abundances are plotted as a function of wavelength in Figure 7. We derive a mean abundance of $\langle\log$ $\varepsilon(\mathrm{Ti})\rangle=4.973 \pm 0.003$ with $\sigma=0.040$; the uncertainties are merely line-to-line scatters. This value is in good agreement with recent compilations of recommended solar photospheric abundances: $4.95 \pm 0.05$ (Asplund et al. 2009) and $4.90 \pm 0.06$ (Lodders et al. 2009).

The "internal" uncertainty in our solar abundance computations is estimated simply by noting that the Ti I line-to-line abundance scatter is just 0.04 and the standard deviation of the abundance mean is virtually zero. More important is the "external" uncertainty, which lies chiefly in the choice of solar 


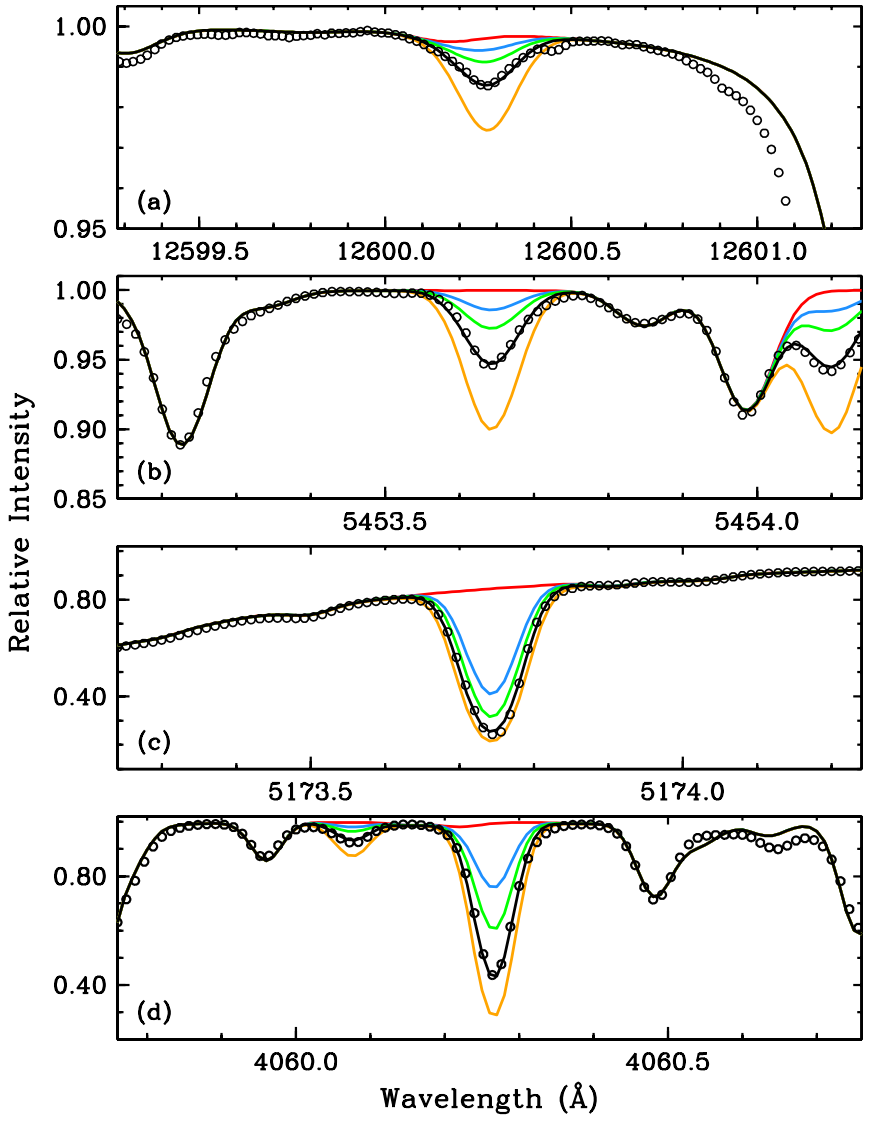

Figure 6. Observed and synthetic spectra of four representative Ti I lines used in our solar photospheric abundance analysis. The points are from the Delbouille et al. (1973) atlas, but for clarity in this figure we plot only every fourth point in panel (a) and every sixth point in the other three panels. The lines in each panel represent five synthetic spectra. The black line is for the "best-fit" Ti abundance for this line reported in Table 4. The orange line is for an abundance 0.3 dex larger, the green line is for an abundance 0.3 dex smaller, and the blue line is for an abundance 0.6 dex smaller than the derived $\mathrm{Ti}$ abundance. The red line is from a synthesis computed without any contribution from Ti. In panel (b) the absorption at 5454.1 $\AA$ is due to Ti II, and in panel (d) the absorption at $4060.1 \AA$ is due to Ti I; neither of these lines are part of the present laboratory study.

(A color version of this figure is available in the online journal.)

Table 4

Solar Photospheric Titanium Abundances from Individual Ti I Lines

\begin{tabular}{lccc}
\hline \hline $\begin{array}{l}\text { Wavelength in Air } \\
(\AA)\end{array}$ & $\begin{array}{c}\text { Lower Energy } \\
(\mathrm{eV})\end{array}$ & $\log _{10}(g f)$ & $\log _{10}(\varepsilon)$ \\
\hline 3199.914 & 0.048 & 0.31 & 4.99 \\
3717.391 & 0.000 & -1.19 & 4.95 \\
3725.152 & 1.066 & -0.34 & 4.89 \\
3729.807 & 0.000 & -0.28 & 4.99 \\
3924.530 & 0.021 & -0.87 & 4.99 \\
\hline
\end{tabular}

(This table is available in its entirety in a machine-readable form in the online journal. A portion is shown here for guidance regarding its form and content.)

photospheric model. We have chosen to adopt the Holweger \& Müller (1974) empirical model to be consistent with all of the previous studies in our lab/solar transition investigation. An alternative is to use a radiative-equilibrium model, and so we have interpolated within the ATLAS grid (Kurucz 2011, and references therein) and run test synthetic spectrum calculations on various Ti I lines. These suggest a smaller photospheric abundance by $\approx 0.1$ dex than we have computed with the Holweger $\&$ Müller model. For the purpose of this paper we elect not to

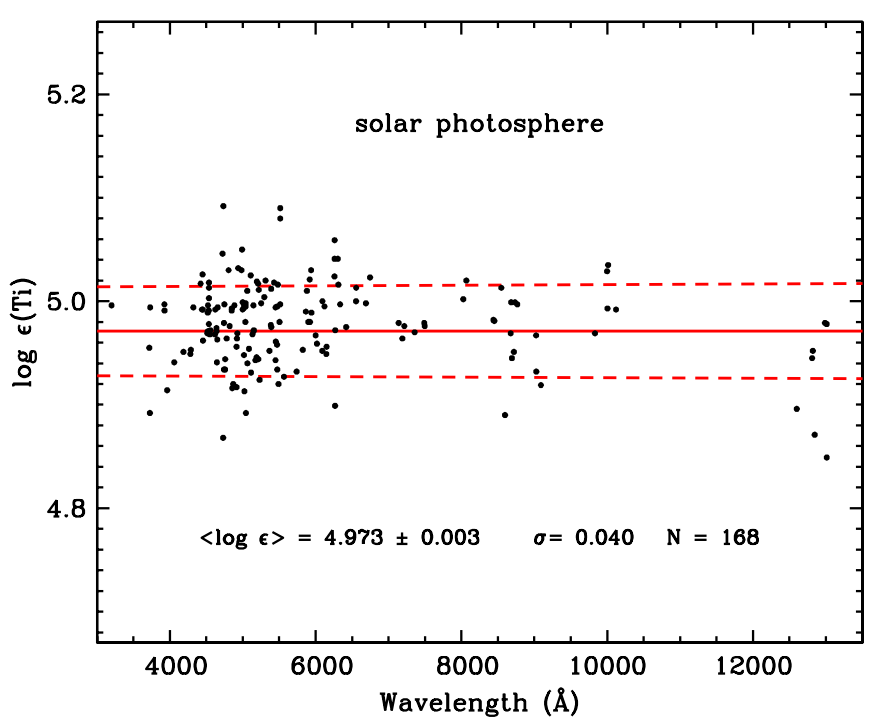

Figure 7. Solar photospheric Ti abundances as a function of wavelength. For display purposes, in this figure we add random "noise" to the abundances with an envelope amplitude of $\leqslant \pm 0.01$, in order to see more clearly the individual points. The abundance statistics written in the figure legend are computed with the original abundance set (Table 4). The red solid line represents the mean Ti abundance, and the red dashed lines are drawn at $\pm 1 \sigma$ from the mean.

(A color version of this figure is available in the online journal.)

average the results between the two solar models. However, it suggests that the true photospheric Ti abundance uncertainties from neutral Ti transitions is perhaps \pm 0.05 ; the abundance scale uncertainties are larger than the internal scatter uncertainties. More detailed solar abundance computations should be performed in the future with these new transition probabilities. In particular, re-consideration of the NLTE computations of Bergemann (2011) and application of three-dimensional solar atmospheric computations to the photospheric Ti abundance problem (as outlined in, e.g., Asplund et al. 2009) would be welcome.

\section{THE TITANIUM ABUNDANCE IN THE PHOTOSPHERE OF HD 84937}

HD 84937 is a bright ${ }^{10}$ metal-poor main-sequence turnoff $\operatorname{star}([\mathrm{Fe} / \mathrm{H}] \approx-2.1$; e.g., Mashonkina et al. 2011, and many earlier studies). Its spectrum has undergone many abundance analyses, beginning with those of Grabowski (1976) and Peterson (1978). HD 84937 is one of the few metal-poor halo stars with a solid detection of ${ }^{6} \mathrm{Li}$ (e.g., Smith et al. 1993; Hobbs \& Thorburn 1997; Cayrel et al. 1999). HD 84937 is a nearby star with a well-determined HIPPARCOS parallax. Lacking significant interstellar extinction, HD 84937's broadband photometry can yield accurate effective temperature estimates. The $V$ magnitude, parallax, and an assumed metal-poor main-sequence turnoff mass can then be used to derive an accurate surface gravity. All atmospheric parameters for HD 84937 can be determined well independent of any spectroscopic information.

\subsection{High-resolution Spectra}

A vacuum-UV spectrum of HD 84937 was obtained with the Space Telescope Imaging Spectrograph (STIS) of the HST, under proposal \#7402 (PI: R. C. Peterson). The spectra formed ${ }_{10}$ Adopted from the SIMBAD database: http://simbad.u-strasbg.fr/simbad/
sim-fid. 
part of a data set to search for chromospheric emission in metalpoor main-sequence stars (Peterson \& Schrijver 1997). The instrumental characteristics are discussed in detail by Peterson et al. (2001). To summarize, STIS was configured in its highresolution mid-UV mode through the use of the E230M grating. This produced spectra covering the wavelength range $2280 \AA \leqslant$ $\lambda \leqslant 3120 \AA$, with an effective resolving power $R \approx 25000$. Two integrations were obtained on HD 84937 with this setup, and we co-add these to increase the effective $\mathrm{S} / \mathrm{N}$. There are few truly absorption-free regions in the UV spectral region even in a warm metal-poor star. However, repeated synthetic/observed spectrum matches suggest that the $\mathrm{S} / \mathrm{N}$ ratios varied from $\approx 30$ at $2300 \AA$ to $\approx 60$ at $3100 \AA$.

Excellent ground-based spectra of HD 84937 were obtained from the ESO VLT UVES archive. ${ }^{11}$ These spectra cover a total wavelength range 3100-10000 $\AA$, have resolving power $R \sim$ 60,000 , and $\mathrm{S} / \mathrm{N} \approx 100$ at $3500 \AA, 200$ at $4500 \AA$, and 300 at $5500 \AA$.

\subsection{Model Atmosphere Parameters}

The many analyses of HD 84937 have derived or adopted model parameters that have very little variation. The SAGA bibliographic database of metal-poor stellar analyses (Suda et al. 2008) ${ }^{12}$ lists more than 20 papers published since 2000 that include HD 84937. Here, we discuss in turn the effective temperature, surface gravity, metallicity, and microturbulent velocity of this star.

Effective temperature: the SAGA database entries yield $\left\langle T_{\text {eff }}\right\rangle=6305 \mathrm{~K}(\sigma=65 \mathrm{~K})$. The SIMBAD ${ }^{13}$ datbase lists $V=8.28$ and $K=7.062$ for HD 84937, or $V-K=1.22$. The HIPPARCOS parallax (van Leeuwen 2007), $\pi=13.74 \pm 0.78$, yields a distance $d=72.8 \pm 4.1 \mathrm{pc}$. This suggests that there is no significant interstellar reddening toward HD 84937. Application of the Alonso et al. (1996) $V-K$ temperature calibration then yields $T_{\text {eff }} \approx 6270 \mathrm{~K}$, and the Ramírez \& Meléndez (2005) calibration yields $T_{\text {eff }} \approx 6380 \mathrm{~K}$. As all of these temperatures are in reasonable agreement, we adopt $T_{\text {eff }} \approx 6300 \mathrm{~K}$ for this paper.

Surface gravity: the SAGA entries average to $\langle\log g\rangle=4.00$ $(\sigma=0.15)$, but some of these are "physical" values based on the accurate parallax. Assuming a metal-poor star turnoff mass $M=0.85 M_{\odot}$, and a bolometric correction, $\mathrm{BC}=-0.19$ (Alonso et al. 1995), the gravity is

$$
\begin{aligned}
\log g= & 0.4\left(M_{V}+B C-M_{\text {bol }_{\odot}}\right)+\log _{g_{\odot}} \\
& +4 \log \left(T_{\text {eff }} / T_{\text {eff }_{\odot}}\right)+\log \left(M / M_{\odot}\right)=4.1
\end{aligned}
$$

which is close to the SAGA literature average. We adopt a final gravity of $\log g=4.0$ for this paper.

Metallicity: the SAGA entries suggest $\langle[\mathrm{Fe} / \mathrm{H}]\rangle-2.15(\sigma=$ $0.15)$. We use this as the initial metallicity value, but in Section 7.4 we derive a new Fe abundance for HD 84937.

Microturbulent velocity: there is more study-to-study variation in this parameter. The SAGA database reports this number in just 12 of the 21 cited papers, for which $\left\langle v_{t}\right\rangle=1.45 \mathrm{~km} \mathrm{~s}^{-1}$ $\left(\sigma=0.40 \mathrm{~km} \mathrm{~s}^{-1}\right)$. We adopt $v_{\mathrm{t}}=1.5 \mathrm{~km} \mathrm{~s}^{-1}$ for our work. Fortunately all $\mathrm{Ti}$ I transitions employed here are relatively weak, and abundances derived from them in HD 84937 have little sensitivity to microturbulent velocity choice.

\footnotetext{
11 Under programs 073.D-0024 (PI: C. Akerman) and 266.D-5655 (Service Mode).

12 Available at http://saga.sci.hokudai.ac.jp/wiki/doku.php.

13 http://simbad.u-strasbg.fr/simbad/
}

From these considerations, we estimate a reasonable model atmosphere for HD 84937 to be $\left(T_{\text {eff }} / \log g / v_{\mathrm{t}} /[\mathrm{Fe} / \mathrm{H}]\right)=$ $(6300 \pm 100 \mathrm{~K} / 4.0 \pm 0.2 / 1.50 \pm 0.25 /-2.15 \pm 0.20)$. We calculate a model atmosphere for HD 84937 with all these parameters, starting with the Kurucz $(2011)^{14}$ model grid and interpolating with software kindly provided by Andy McWilliam and Inese Ivans.

\subsection{Determination of the Ti Abundance of HD 84937}

We follow the same procedure for the derivation of the $\mathrm{Ti}$ abundance of HD 84937 as we have for the Sun. We first search for suitable lines for analysis, but the metallicity of HD 84937 is more than two orders of magnitude less than that of the Sun. Line selection is thus greatly simplified by initial examinations of Ti I lines in the spectra, which suggests that lines with STR < -1.5 will be undetectably weak in HD 84937 . On the other hand, this general spectrum absorption depth weakness opens up the line-rich near-UV (3000-4000 $)$ and vacuum-UV ( $<3000 \AA)$ spectral regions for Ti abundance analysis. Therefore, while we are unable to use any of the weaker Ti I lines longward of $5200 \AA$ in our VLT UVES spectra, we are able to detect usable Ti I lines with wavelengths as small as $2644 \AA$ in our HST STIS spectrum.

We derive $\mathrm{Ti}$ abundances in HD 84937 from observed/ synthetic spectrum matches for HD 84937 in the same manner as described above for the solar photosphere. However, we generate the synthetic spectra for this very metal-poor star with the enhancement to the synthesis code that includes a more correct treatment of hydrogen Rayleigh scattering. The importance of Rayleigh scattering increases rapidly toward smaller wavelengths. At high stellar metallicities the $\mathrm{H}^{-}$continuum opacity is of course dominant in most stars. However, the $\mathrm{H}^{-}$ opacity weakens in low-metallicity cool stars due to the depletion of the free electron supply (which comes largely from ionization of abundant neutral metals such as $\mathrm{Mg}, \mathrm{Si}$, and $\mathrm{Fe}$ ). Rayleigh scattering, which has no metallicity dependence, can dominate over $\mathrm{H}^{-}$at wavelengths $\lambda \leqslant 4500 \AA$ in cooler stars $\left(T_{\text {eff }} \leqslant 5000 \mathrm{~K}\right)$ for metallicities $[\mathrm{Fe} / \mathrm{H}] \leqslant-1.5$. This is a special concern for abundance analyses of metal-poor red giants, as emphasized by Cayrel et al. (2004). This effect is much less of a concern in stars with $T_{\text {eff }}>6000 \mathrm{~K}$, because in these warmer atmospheres the electrons for $\mathrm{H}^{-}$come largely from $\mathrm{H}$ i itself, and the $\mathrm{H}^{-}$opacity is larger than that of $\mathrm{H}$ I Rayleigh scattering even at wavelengths in the vacuum UV. Nevertheless, we compute synthetic spectra for HD 84937 with realistic Rayleigh scattering formulae, source functions, and radiative transfer solutions that drop the standard LTE integral calculations that do not distinguish between pure absorption and scattering in the continuum. This version of the spectrum code is described by Sobeck et al. (2011).

In Figure 8, we show four representative $\mathrm{Ti}$ I features in HD 84937. Panel (a) displays synthetic and observed spectra of the $5173.7 \AA$ line, one of the longest-wavelength Ti I transitions detected on our spectra. It can be compared to the same line in the solar spectrum shown in Figure 6(c). Here the overall metal deficiency weakens all absorption features, so the sloping continuum due to the $\mathrm{Mg}$ I $\mathrm{b}$ line in the solar spectrum becomes a small depression mostly blueward of the very weak $(\leqslant 2 \%)$ Ti I absorption. Panel (b) contains the $4305.9 \AA$ Ti I line that is usually overwhelmed by strong absorption from the $\mathrm{CH}$ $G$ band. In HD 84937, the $\mathrm{CH}$ lines are very weak in this small spectral window contributing only to detectable absorptions

\footnotetext{
14 http://kurucz.harvard.edu/grids.html
} 

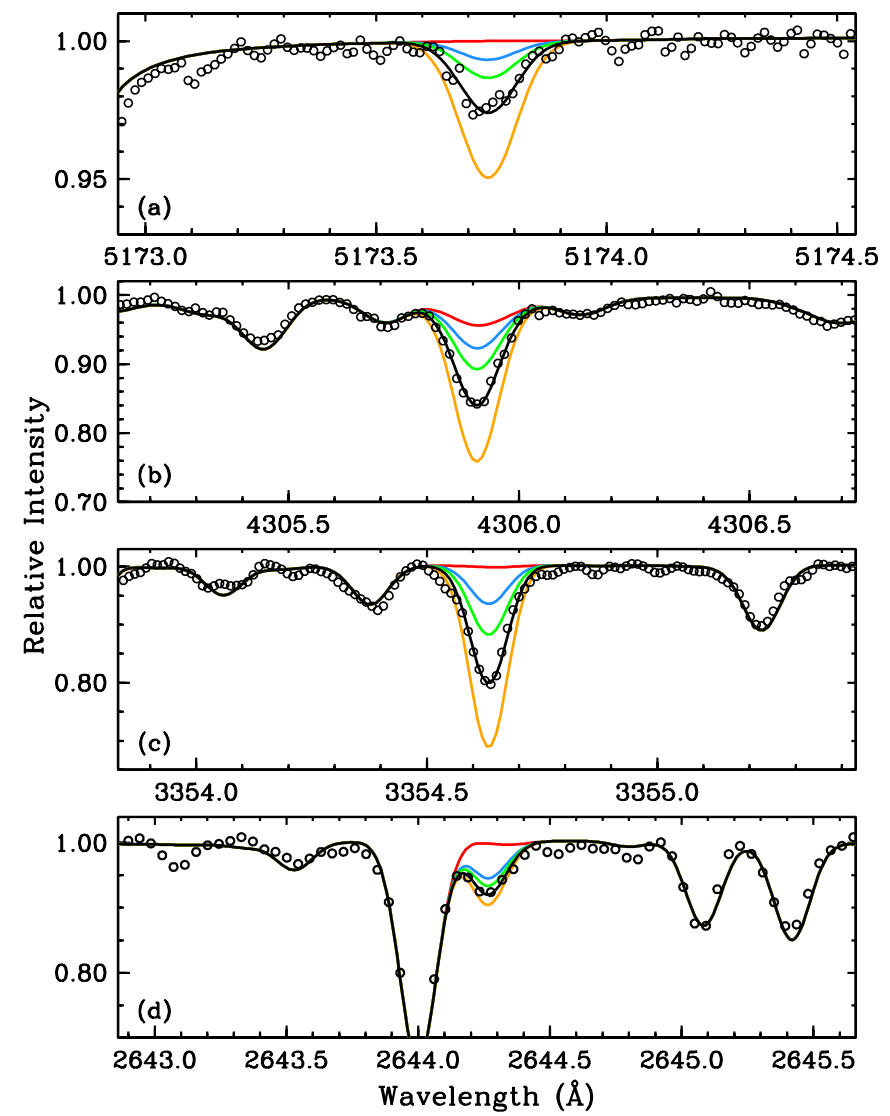

Figure 8. Observed and synthetic spectra of four representative Ti I lines used in our HD 84937 abundance analysis. The points are from the observed spectra described in Section 7.1. The lines in each panel represent five synthetic spectra. The black line is for the "best-fit" Ti abundance for this line reported in Table 5. The orange line is for an abundance 0.3 dex larger, the green line is for an abundance 0.3 dex smaller, and the blue line is for an abundance 0.6 dex smaller than the derived Ti abundance. The red line is from a synthesis computed without any contribution from $\mathrm{Ti}$.

(A color version of this figure is available in the online journal.)

at 4305.3 and $4306.7 \AA$, to a portion of $4306.1 \AA$, and to the non-Ti part of $4304.9 \AA$. The features at 4305.4 and $4305.7 \AA$ arise from other Fe-peak species. The Ti I line is clearly the dominant feature in this small spectral interval. Panel (c) shows a Ti I line in the crowded near-UV spectral region that features the NH bandheads near 3360 and $3370 \AA$, but Moore et al. (1966) do not identify any NH lines in the solar spectrum in the wavelength range of this panel. Finally, panel (d) displays one of two lines that we were able to use for Ti abundance computations in the vacuum-UV spectral region.

Ti abundances derived from individual Ti I lines are given in Table 5, and plotted as a function of wavelength in Figure 9. From a final list of 54 suitable Ti I lines in HD 84937 we derive $\langle\log \varepsilon(\mathrm{Ti})\rangle=3.122 \pm 0.007(\sigma=0.054)$. The quoted uncertainties are statistical "scatter" errors only. The relative Ti abundance is thus $[\mathrm{Ti} / \mathrm{H}]=3.122-4.973=-1.851$. There are 23 Ti I lines in common between our analyses of HD 84937 and the Sun. For just these transitions we find $\langle[\mathrm{Ti} / \mathrm{H}]\rangle=$ $-1.842 \pm 0.013(\sigma=0.062)$, in excellent agreement with the Ti relative abundance computed from the mean values in $\mathrm{HD}$ 84937 and the Sun.

\subsection{The Relative Ti Enhancement in HD 84937}

In metal-poor stars Ti has been long known to have $\alpha$-elementlike abundance enhancements, i.e., $[\mathrm{Ti} / \mathrm{Fe}] \sim+0.2$ to +0.5 . Our

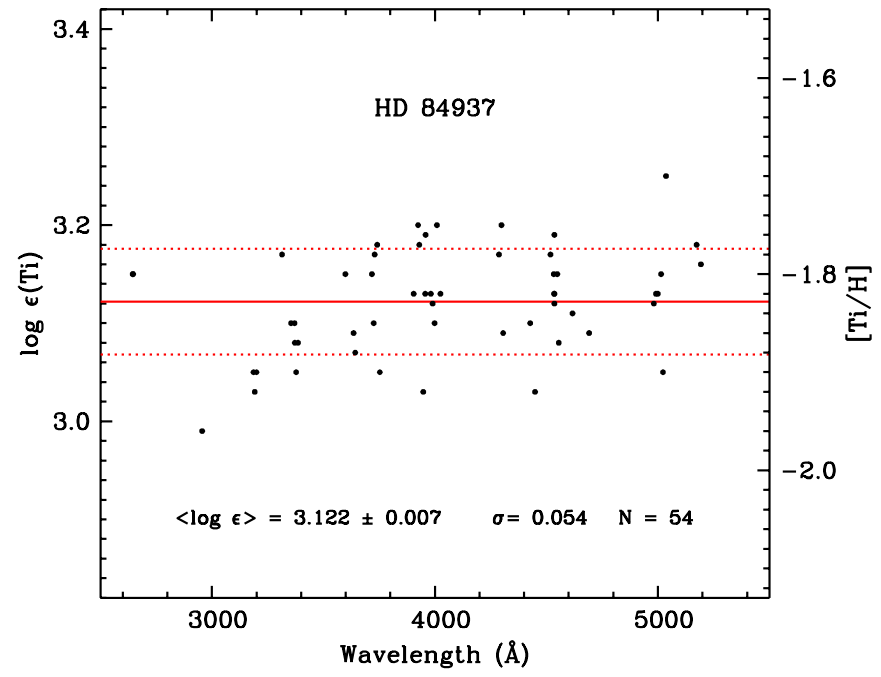

Figure 9. Ti abundances in HD 84937 as a function of wavelength. The red solid line represents the mean Ti abundance, and the red dashed lines are drawn at $\pm 1 \sigma$ from the mean. The relative $[\mathrm{Ti} / \mathrm{H}]$ values marked on the right vertical axis are computed with the assumption that $\log \varepsilon(\mathrm{Ti})_{\odot}=4.95$ (Asplund et al. 2009).

(A color version of this figure is available in the online journal.)

Table 5

HD 84937 Titanium Abundances from Individual Ti I Lines

\begin{tabular}{lccc}
\hline \hline $\begin{array}{l}\text { Wavelength in Air } \\
(\AA)\end{array}$ & $\begin{array}{c}\text { Lower Energy } \\
(\mathrm{eV})\end{array}$ & $\log _{10}(g f)$ & $\log _{10}(\varepsilon)$ \\
\hline 2644.263 & 0.021 & -0.08 & 3.15 \\
2646.634 & 0.048 & +0.06 & 3.15 \\
2956.132 & 0.048 & +0.12 & 2.99 \\
3186.451 & 0.000 & +0.01 & 3.05 \\
3191.992 & 0.021 & +0.16 & 3.03 \\
\hline
\end{tabular}

(This table is available in its entirety in a machine-readable form in the online journal. A portion is shown here for guidance regarding its form and content.)

new Ti abundance for HD 84937 ought to be accompanied by a new $\mathrm{Fe}$ abundance to produce the most accurate $[\mathrm{Ti} / \mathrm{Fe}]$. There are a large number of $\mathrm{Fe}$ I lines visible on our spectra of this star. Unfortunately there is no single source of laboratory transition probabilities that can be applied here. Therefore, we choose to adopt Fe I $g f$-values from the "critical compilation" of Fuhr \& Wiese (2006). ${ }^{15}$ Those authors estimate the quality of each transition probability included in their compilation, and we chose to include only Fe I lines with quality grade of "C" or better. ${ }^{16}$ To determine the $\mathrm{Fe}$ abundance of HD 84937 we go through an identical procedure for these Fe I lines that we have done for Ti I: identify the detectable unblended lines, compute synthetic spectra, and compare to the observed features.

In Figure 10, we show the results of the new Fe abundance calculations. From $421 \mathrm{Fe}$ I lines in the wavelength range $2300 \AA<\lambda<6700 \AA$ we derive $\langle\log \varepsilon(\mathrm{Fe})\rangle=5.180 \pm 0.004$ $(\sigma=0.081)$. Assuming the $\log \varepsilon(\mathrm{Fe})_{\odot}=7.50 \pm 0.04$ leads to an Fe-metallicity of $[\mathrm{Fe} / \mathrm{H}]=5.18-7.50=-2.32$. A comparison of the $\mathrm{Ti}$ and $\mathrm{Fe}$ abundances then yields $[\mathrm{Ti} / \mathrm{Fe}]=-1.85+$ $2.32=+0.47$.

However, the data of Figure 10 clearly reveal some small Fe abundance trends with wavelength. In particular, in the near-UV

\footnotetext{
$\overline{15}$ Most of these data are available at http://www.nist.gov/pml/data/asd.cfm.

16 Their estimates of minimum accuracies for each grade of interest here are: $\mathrm{A}, \leqslant 3 \% ; \mathrm{B}, \leqslant 10 \% ; \mathrm{C}, \leqslant 25 \% ; \mathrm{D}, \leqslant 50 \%$; and $\mathrm{E},>50 \%$.
} 


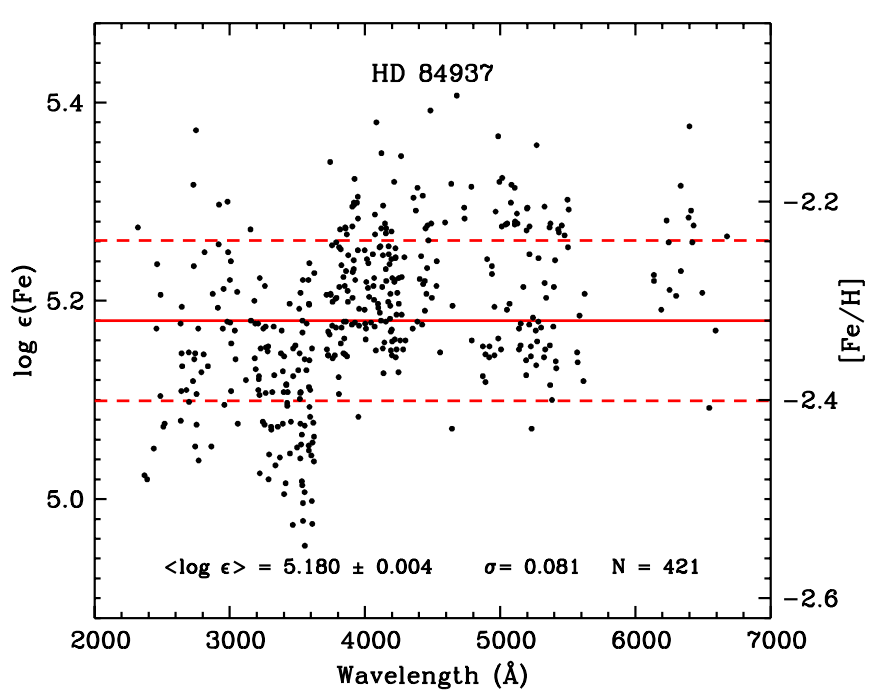

Figure 10. Fe abundances in HD 84937 as a function of wavelength. For display purposes, in this figure we add random "noise" to the abundances with an envelope amplitude of $\leqslant \pm 0.01$, in order to see more clearly the individual points. The red solid line represents the mean Fe abundance, and the red dashed lines are drawn at $\pm 1 \sigma$ from the mean. The relative $[\mathrm{Fe} / \mathrm{H}]$ values marked on the right vertical axis are computed with the assumption that $\log \varepsilon(\mathrm{Fe})_{\odot}=7.50$ (Asplund et al. 2009).

(A color version of this figure is available in the online journal.)

spectral region $(3100 \AA<\lambda<3700 \AA)$ we derive $\langle\log \varepsilon(\mathrm{Fe})\rangle=$ $5.104 \pm 0.007(\sigma=0.067,101$ lines $)$, compared with the mean abundance at longer wavelengths $(\langle\log \varepsilon(\mathrm{Fe})\rangle=5.214 \pm 0.004$ ( $\sigma=0.062,267$ lines $)$. The mean in the vacuum-UV $(2300 \AA<$ $\lambda<3100 \AA$ ) splits the difference: $\langle\log \varepsilon(\mathrm{Fe})\rangle=5.156 \pm 0.011$ ( $\sigma=0.077,53$ lines). The abundance depression in the near-UV has also been found recently in several other metal-poor stars (Roederer et al. 2012; see their Figure 10). That paper argues that the problem is unlikely to lie in departures from LTE in the populations of energy levels of Fe I. A more likely culprit is an underestimation of the $\mathrm{H}_{\mathrm{I}}$ continuum opacity beginning at the Balmer $n=2$ ionization limit $(3647 \AA)$. In Figure 11 of Roederer et al. the relative contributions of $\mathrm{H}^{-}, \mathrm{H}$ I bound-free, and Hi Rayleigh scattering to the total opacity in the warm metal-poor giant HD $108317\left(T_{\text {eff }}=5100 \mathrm{~K}, \log g=2.67\right.$, $[\mathrm{Fe} / \mathrm{H}]=-2.37)$ are shown. $\mathrm{H}^{-}$opacity dominates over Rayeigh scattering at all wavelengths for this star, but the $\mathrm{H}$ I Balmer continuum opacity nearly equals the $\mathrm{H}^{-}$opacity for $\lambda<3647 \AA$. Similar calculations for HD $84937\left(T_{\text {eff }}=\right.$ $6300 \mathrm{~K}, \log g=4.00,[\mathrm{Fe} / \mathrm{H}]=-2.32$ ) yield the same trends with wavelength and roughly equal amounts of $\mathrm{H}^{-}$and $\mathrm{HI}_{\mathrm{I}}$ to the continuous opacity in the near-UV spectral region. The fractional contribution of $\mathrm{H}$ I to the total opacity diminishes in the vacuum-UV. Roederer et al. were unable to identify the cause of the Fe abundance anomaly in the near-UV; we suppose that it may involve departures from LTE in the $n=2$ state population of $\mathrm{HI}$, but have no further empirical evidence to shed light on this issue.

A hint of the same effect is seen in our Ti I results, although we do not have many lines of this species in the near-UV region. For 39 lines with $\lambda>3700 \AA$, the mean abundance is $\langle\log \varepsilon(\mathrm{Ti})\rangle=3.135 \pm 0.008(\sigma=0.050)$, and for 15 lines at smaller wavelengths, $\langle\log \varepsilon(\mathrm{Ti})\rangle=3.087 \pm 0.013(\sigma=0.051)$. Forming relative abundances in the usual way, for $\lambda>3700 \AA$ we calculate $[\mathrm{Ti} / \mathrm{Fe}]=+0.45$, in good agreement with the +0.47 value obtained with the entire sets of $\mathrm{Fe}$ and $\mathrm{Ti}$ lines.

\subsection{Abundance Uncertainties}

The internal line-to-line scatter uncertainties, which combine continuum placement errors and line profile fitting errors, are estimated to be $\leqslant 0.04$ dex. For external scale errors we re-computed abundances of some lines using different model atmosphere parameters. First considering Ti I: for $\Delta\left(T_{\text {eff }}\right)=$ $+150 \mathrm{~K}, \Delta(\log \varepsilon) \approx+0.12$; for $\Delta(\log g)=+0.3, \Delta(\log \varepsilon) \approx$ -0.01 ; for $\Delta([\mathrm{M} / \mathrm{H}])=-0.3, \Delta(\log \varepsilon) \approx-0.01$; and $\Delta\left(v_{t}\right)=$ $-0.25, \Delta(\log \varepsilon)<0.05$ for the weak lines employed in this study. The sensitivity to temperature changes but not to gravity changes is understandable in terms of the Saha ionization equilibria for both $\mathrm{Ti} \mathrm{I}$ and $\mathrm{H}^{-}$(the dominant continuous opacity source in most spectral regions). The $\mathrm{H}^{-}$dominance also explains the lack of change in $\mathrm{Ti}$ abundance with changes in model metallicity. Finally, since most of the Ti I lines are weak, changes in $v_{t}$ are not important Ti abundance error sources here. More importantly, the Fe I transitions discussed in Section 7.4 have nearly the same abundance responses to model parameter uncertainties. Thus, the relative abundance ratios $[\mathrm{Ti} / \mathrm{Fe}]$ are nearly insensitive to $T_{\text {eff }}, \log g,[\mathrm{M} / \mathrm{H}]$, and $v_{t}$ changes.

Bergemann (2011) has investigated non-LTE line formation in the Sun and in several metal-poor stars, including HD 84937. Using a similar set of model parameters as we have, but generating a model atmosphere with a different code than that of Kurucz (2011), she computes [Ti $/ \mathrm{Fe}]_{\mathrm{LTE}}=+0.49$, very close to our result. Accounting for departures from LTE results in a larger relative abundance, $[\mathrm{Ti} \mathrm{I} / \mathrm{Fe}]_{\mathrm{NLTE}}=+0.63$. Bergemann's calculations for ionized transitions yields $[\mathrm{Ti} \text { II } / \mathrm{Fe}]_{\mathrm{LTE}}=$ $[\mathrm{Ti} \text { II } / \mathrm{Fe}]_{\mathrm{NLTE}}=+0.34$. She suggests the discordance between the neutral and ion abundances indicates that the NLTE abundances are overestimated for high-gravity metal-poor stars such as HD 84937, and that reliable abundances are best obtained with ionized-species transitions. In Wood et al. (2013), we will consider this question again with new transition probabilities for Ti II lines.

\section{IMPLICATIONS FOR STELLAR NUCLEOSYNTHESIS}

Titanium is an Fe-peak element formed in core collapse supernovae (Thielemann et al. 1996). The abundance of this element with respect to $\mathrm{Fe}$ and other $\mathrm{Fe}$-peak elements provides a signature of the type of synthesis conditions in the exploding star. Specifically, in very evolved massive stars these types of elements are produced in explosive Si burning, either complete or incomplete. How the various Fe-peak elements are formed depends critically upon such supernova parameters as where the mass cut is located (the point in the star above which the mass is ejected), the neutron excess, explosion energy, and supernova progenitor mass (Nakamura et al. 1999). Determining these parameters in a precise manner, using the abundances as a constraint for the model calculations, provides direct insight into exactly how supernovae explode.

We have measured the $\mathrm{Ti}$ and $\mathrm{Fe}$ abundances in the halo star HD 84937 and in the solar photosphere, deriving $[\mathrm{Ti} / \mathrm{Fe}]=+0.47$ for HD 84397 (Section 7.4). This result, like previous studies that have yielded substantial Ti overabundances in metal-poor stars, presents a puzzle for nucleosynthesis predictions. The main isotope (74\%) of $\mathrm{Ti}$ is ${ }^{48} \mathrm{Ti}$, produced initially in core collapse supernova nucleosynthesis as ${ }^{48} \mathrm{Cr}$, along with ${ }^{56} \mathrm{Ni}$ which decays to ${ }^{56} \mathrm{Fe}$ (92\% of elemental $\left.\mathrm{Fe}\right)$. Employing our value of $[\mathrm{Ti} / \mathrm{Fe}]=+0.47$ along with the solar abundance values for $\mathrm{Ti}$ and $\mathrm{Fe}$ gives a value of abundances, $Y$, of these isotopes as $\log _{10}\left(Y_{48} / Y_{56}\right)=0.47-7.46+4.93=-2.06$. 
Model abundances are frequently given in mass fractions $X$, where $Y=X / A$ and $A$ is the atomic weight. Thus, one would expect the mass fraction ratio in the case of the isotopes that were injected into HD 84397 to be approximately $\log _{10}\left(X_{48} / X_{56}\right)=-2.06-\log (56 / 48)=-2.13$ or $X_{48} / X_{56}=$ $7.4 \mathrm{E}-03$. This is an approximation since ${ }^{48} \mathrm{Ti}$ does not represent the total $\mathrm{Ti}$ elemental abundance and one needs to add some smaller contribution from $\mathrm{Cr}$ isotopes to obtain the total Ti abundance values. Nevertheless, comparison of this calculated ratio with the literature indicates that supernova models (e.g., Thielemann et al. 1996; Heger \& Woosley 2002), as well as hypernova models (Nomoto et al. 2006), consistently under produce the amount of observed $\mathrm{Ti}$ in the metal-poor halo stars. Therefore, the new and more precise Ti value for HD 84937, and the derived $[\mathrm{Ti} / \mathrm{Fe}]$ value, will provide important constraints on models of supernovae explosions and nucleosynthesis.

In addition to individual nucleosynthetic yields the Fe-peak abundances provide direct insight into Galactic chemical evolution (see, e.g., McWilliam et al. 1995a, 1995b; Timmes et al. 1995; McWilliam 1997; François et al. 2004; Henry et al. 2010). These studies have found a general increase in the $[\mathrm{Ti} / \mathrm{Fe}]$ value from solar to $[\mathrm{Ti} / \mathrm{Fe}] \sim=+0.4$ going from a metallicity of solar to approximately $[\mathrm{Fe} / \mathrm{H}]=-1$. For $[\mathrm{Fe} / \mathrm{H}]<-1$ the $[\mathrm{Ti} / \mathrm{Fe}]$ value remains near +0.4 but with large scatter at the very low metallicity range near $[\mathrm{Fe} / \mathrm{H}]=-3$ (Henry et al. 2010). Our new experimental value for [Ti/Fe] in HD 84397 is consistent with, but falls on the high side of the general trend line of, other metal-poor halo stars. These abundance trends in Fe-peak elements versus metallicity are significant because they provide direct constraints on massive star yields, something that is not yet well defined.

\section{SUMMARY}

New branching fraction measurements for 948 lines of Ti I from archived FTS spectra and new data recorded with a highresolution echelle spectrometer are reported. The branching fractions are combined with published radiative lifetimes from LIF measurements to determine accurate, absolute atomic transition probabilities for 948 lines of Ti I. Generally good agreement is found in comparisons of these new data to data from the NIST Atomic Spectra Database. The new data are applied to re-determine the $\mathrm{Ti}$ abundance in the photospheres of the Sun and metal-poor star HD 84937 using many lines covering a range of wavelength and E.P. values. Abundance values from Ti I lines have small scatter and small wavelength dependence in HD 84937. However, this Ti study yields some evidence for extra photospheric continuum opacity near the Balmer limit. Similar effects are seen using Fe I lines in this study of HD 84937 and previously in studies of other metal-poor stars. The variation of relative $\mathrm{Ti} / \mathrm{Fe}$ abundance with metallicity in metal-poor stars, as observed in numerous earlier studies, is supported in this study. Supernova models consistently underproduce the amount of $\mathrm{Ti}$ seen in metal-poor stars. Relative abundance trends of Fe-group elements as a function of metallicity provide direct constraints on massive star yields.

We thank F.-K. Thielemann for helpful discussions on supernova production of $\mathrm{Fe}$ and $\mathrm{Ti}$. This work is supported in part by NSF grant AST-1211055 (J.E.L.), NSF REU grant AST-1004881 (A.G.), and NSF grants AST-0908978, and AST-1211585 (C.S.). Some data for this project were obtained from the ESO Science Archive Facility under request numbers 073.D-0024 (PI: C. Akerman) and 266.D-5655 (Service Mode).
Other data were from observations made with the NASA/ESA Hubble Space Telescope, obtained from the data archive at the Space Telescope Science Institute. STScI is operated by the Association of Universities for Research in Astronomy, Inc. under NASA contract NAS 5-26555. The solar spectra were acquired from the BASS2000 solar data archive. We thank J. S. Sobeck for helpful discussions.

\section{REFERENCES}

Adams, D. L., \& Whaling, W. 1981, JOSA, 71, 1036

Alonso, A., Arribas, S., \& Martínez-Roger, C. 1995, A\&A, 297, 197

Alonso, A., Arribas, S., \& Martínez-Roger, C. 1996, A\&A, 313, 873

Asplund, M. 2005, ARA\&A, 43, 481

Asplund, M., Grevesse, N., Sauval, A. J., \& Scott, P. 2009, ARA\&A, 47, 481

Barklem, P. S., Christlieb, N., Beers, T. C., et al. 2005, A\&A, 439, 129

Bell, G. D., Kalman, L. B., \& Tubbs, E. F. 1975, ApJ, 200, 520

Bergemann, M. 2011, A\&A, 413, 2184

Bergemann, M., \& Gehren, T. 2007, A\&A, 473, 291

Bergemann, M., \& Gehren, T. 2008, A\&A, 492, 823

Bergeson, S. D., Mullman, K. L., \& Lawler, J. E. 1996, ApJ, 464, 1050

Bíemont, É., Blagoev, K., Engström, L., et al. 2011, MNRAS, 414, 3350

Bizzarri, A., Huber, M. C. E., Noels, A., et al. 1993, A\&A, 273, 707

Blackwell, D. E., Booth, A. J., Menon, S. L. R., \& Petford, A. D. 1986, MNRAS, 220, 289

Blackwell, D. E., Menon, S. L. R., \& Petford, A. D. 1983, MNRAS, 204, 883

Blackwell, D. E., Menon, S. L. R., Petford, A. D., \& Shallis, M. J. 1982b, MNRAS, 201, 611

Blackwell, D. E., Petford, A. D., Shallis, M. J., \& Leggett, S. 1982a, MNRAS, 199,21

Blackwell-Whitehead, R. J., Lundberg, H., Nave, G., et al. 2006, MNRAS, 373, 1603

Brault, J. W. 1976, JOSA, 66, 1081

Cayrel, R., Depagne, E., Spite, M., et al. 2004, A\&A, 416, 1117

Cayrel, R., Spite, M., Spite, F., et al. 1999, A\&A, 343, 923

Cowan, J. J., Sneden, C., Burles, S., et al. 2002, ApJ, 572, 861

Danzmann, K., \& Kock, M. 1982, JOSA, 72, 1556

Delbouille, L, Roland, G., \& Neven, L. 1973, Photometric Atlas of the Solar Spectrum from $\lambda 3000$ to $\lambda 10000$ (Liège: Inst. d'Ap., Univ. de Liège)

Den Hartog, E. A., Lawler, J. E., Sobeck, J. S., Sneden, C., \& Cowan, J. J. 2011, ApJS, 194, 35

François, P., Matteucci, F., Cayrel, R., et al. 2004, A\&A, 421, 613

Fuhr, J. R., \& Wiese, W. L. 2006, JPCRD, 35, 1669

Grabowski, B. 1976, AcA, 26, 147

Grevesse, N., Blackwell, D. E., \& Petford, A. D. 1989, A\&A, 208, 157

Hashiguchi, S., \& Hasikuni, M. 1985, JPSJ, 54, 1290

Heger, A., \& Woosley, S. E. 2002, ApJ, 567, 532

Henry, R. B. C., Cowan, J. J., \& Sobeck, J. 2010, ApJ, 709, 715

Hobbs, L. M., \& Thorburn, J. A. 1997, ApJ, 491, 772

Holweger, H., \& Müller, E. A. 1974, SoPh, 39, 19

Holys, A., \& Fuhr, J. R. 1980, A\&A, 90, 14

Kostyk, R. I. 1982, AZh, 59, 693 (Engl. trans., 1982, SvA, 26, 422)

Kühne, M., Danzmann, K., \& Kock, M. 1978, A\&A, 64, 111

Kurucz, R. L. 2011, CaJPh, 89, 417

Lawler, J. E. 1991, A\&A, 252, 853

Lawler, J. E., Bergeson, S. D., Fedchak, J. A., \& Mullman, K. L. 1999, PhyS, T83, 11

Lawler, J. E., Sneden, C., Cowan, J. J., Ivans, I. I., \& Den Hartog, E. A. 2009, ApJS, 182, 51

Lodders, K., Palme, H., \& Gail, H.-P. 2009, Landolt-Börnstein, 4B, 44L

Lotrian, J., Cariou, J., \& Johannin-Gilles, A. 1975, JQSRT, 15, 815

Malcheva, G., Blagoev, K., Mayo, R., et al. 2006, MNRAS, 367, 754

Martin, G. A., Fuhr, J. R., \& Wiese, W. L. 1988, JPCRD, 17, 85

Mashonkina, L., Gehren, T., Shi, J.-R., Korn, A. J., \& Grupp, F. 2011, A\&A, 528, A87

McWilliam, A. 1997, ARA\&A, 35, 503

McWilliam, A., Preston, G. W., Sneden, C., \& Searle, L. 1995a, AJ, 109,2757

McWilliam, A., Preston, G. W., Sneden, C., \& Shectman, S. 1995b, AJ, 109,2736

Moore, C. E., Minnaert, M. G. J., \& Houtgast, J. 1966, The Solar Spectrum $2935 \AA$ to $8770 \AA$ (NBS Monograph Vol. 61; Washington, DC: US GPO)

Morton, D. C. 2003, ApJS, 149, 205 (erratum ApJS, 151, 403 [2004])

Nakamura, T., Umeda, H., Nomoto, K., Thielemann, F.-K., \& Burrows, A. 1999, ApJ, 517, 193 
Nitz, D. E., Wickliffe, M. E., \& Lawler, J. E. 1998, ApJS, 117, 313

Nomoto, K., Tominaga, N., Umeda, H., Kobayashi, C., \& Maeda, K. 2006, $\mathrm{NuPhA}, 777,424$

Peck, E. R., \& Reeder, K. 1972, JOSA, 62, 958

Peterson, R. C. 1978, ApJ, 222, 181

Peterson, R. C., Dorman, B., \& Rood, R. T. 2001, ApJ, 559, 372

Peterson, R. C., \& Schrijver, C. J. 1997, ApJL, 480, L47

Pickering, J. C., Thorne, A. P., \& Perez, R. 2001, ApJS, 132, 403 (erratum ApJS, 138, 247 [2002])

Ramírez, I., \& Meléndez, J. 2005, ApJ, 626, 446

Roberts, J. R., Andersen, T., \& Sorensen, G. 1973, ApJ, 181, 567

Roberts, J. R., Voigt, P. A., \& Czernichowski, A. 1975, ApJ, 197, 791

Roederer, I. U., Lawler, J. E., Sobeck, J. S., et al. 2012, ApJS, 203, 27

Rudolph, J., \& Helbig, V. 1982, JPhB, 15, L599

Salih, S., \& Lawler, J. E. 1990, A\&A, 239, 407

Saloman, E. B. 2012, JPCRD, 41, 013101

Smith, P. L., \& Kühne, M. 1978, RSPSA, 362, 263
Smith, V. V., Lambert, D. L., \& Nissen, P. E. 1993, ApJ, 408, 262

Sneden, C. 1973, ApJ, 184, 839

Sneden, C., Cowan, J. J., Lawler, J. E., et al. 2003, ApJ, 591, 936

Sneden, C., Lawler, J. E., Cowan, J. J., Ivans, I. I., \& Den Hartog, E. A. 2009, ApJS, 182, 80

Sobeck, J. S., Kraft, R. P., Sneden, C., et al. 2011, AJ, 141, 175

Sobeck, J. S., Lawler, J. E., \& Sneden, C. 2007, ApJ, 667, 1267

Suda, T., Katsuta, Y., Yamada, S., et al. 2008, PASJ, 60, 1159

Thielemann, F.-K., Nomoto, K., \& Hashimoto, M.-A. 1996, ApJ, 460, 408

Timmes, F. X., Woosley, S. E., \& Weaver, T. A. 1995, ApJS, 98, 617

van Leeuwen, F. 2007, A\&A, 474, 653

Whaling, W., Carle, M. T., \& Pitt, M. L. 1993, JQSRT, 50, 7

Whaling, W., Scalo, J. M., \& Testerman, L. 1977, ApJ, 212, 581

Westin, J., Sneden, C., Gustafsson, B., \& Cowan, J. J. 2000, ApJ, 530, 783

Wickliffe, M. E., Lawler, J. E., \& Nave, G. 2000, JQSRT, 66, 363

Wood, M. P., \& Lawler, J. E. 2012, ApOpt, 51, 8407

Wood, M. P., Lawler, J. E., Sneden, C., \& Cowan, J. J. 2013, ApJ, submitted 